\title{
LOOKING OUT FOR FROBENIUS SUMMANDS ON A BLOWN-UP SURFACE OF $\mathbb{P}^{2}$
}

\author{
NOBUO HARA
}

\begin{abstract}
For an algebraic variety $X$ in characteristic $p>0$, the push-forward $F_{*}^{e} \mathcal{O}_{X}$ of the structure sheaf by an iterated Frobenius endomorphism $F^{e}$ is closely related to the geometry of $X$. We study the decomposition of $F_{*}^{e} \mathcal{O}_{X}$ into direct summands when $X$ is obtained by blowing up the projective plane $\mathbb{P}^{2}$ at four points in general position. We explicitly describe the decomposition of $F_{*}^{e} \mathcal{O}_{X}$ and show that there appear only finitely many direct summands up to isomorphism, when $e$ runs over all positive integers. We also prove that these summands generate the derived category $D^{b}(X)$. On the other hand, we show that there appear infinitely many distinct indecomposable summands of iterated Frobenius push-forwards on a ten-point blowup of $\mathbb{P}^{2}$.
\end{abstract}

Throughout this paper, we work over an algebraically closed field $k$ of characteristic $p>0$. Let $X$ be a smooth variety over $k$ with the Frobenius morphism $F: X \rightarrow X$. Then the push-forward $F_{*}^{e} \mathcal{O}_{X}$ of the structure sheaf of $X$ by the $e$-times iterate of the Frobenius is a locally free sheaf of rank $p^{e \operatorname{dim} X}$. When $X$ is a projective curve, it is known that the structure of the vector bundle $F_{*}^{e} \mathcal{O}_{X}$ heavily depends on the genus $g$ of $X$, as follows.

(1) If $X=\mathbb{P}^{1}$, then $F_{*}^{e} \mathcal{O}_{X} \cong \mathcal{O}_{\mathbb{P}^{1}} \oplus \mathcal{O}_{\mathbb{P}^{1}}(-1)^{\oplus p^{e}-1}$.

(2) If $X$ is an elliptic curve, then $F_{*}^{e} \mathcal{O}_{X}$ is semi-stable, and

(i) if $X$ is ordinary, $F_{*}^{e} \mathcal{O}_{X}$ splits into $p^{e}$ non-isomorphic $p^{e}$-torsion line bundles;

(ii) if $X$ is supersingular, $F_{*}^{e} \mathcal{O}_{X}$ is isomorphic to Atiyah's indecomposable vector bundle $\mathcal{F}_{p^{e}}$ of degree zero and rank $p^{e}([\mathrm{At}])$.

(3) If $g \geq 2$, then $F_{*}^{e} \mathcal{O}_{X}$ is a stable vector bundle (Mehta-Pauly [MP]).

Received March 2, 2015; received in final form November 23, 2015.

This work is partially supported by Grant-in-Aid for Scientific Research, JSPS.

2010 Mathematics Subject Classification. Primary 14J60. Secondary 14G17, 14J26. 
Putting the detailed study of the case of genus $g \geq 2$ aside, the structure of the vector bundle $F_{*}^{e} \mathcal{O}_{X}$ is fairly well-understood in dimension one. So it is natural to ask about the surface case. In general, the Frobenius push-forward $F_{*}^{e} \mathcal{O}_{X}$ of a toric variety $X$ splits into line bundles, and there are only finitely many isomorphism classes of line bundles that appear as a direct summand of $F_{*}^{e} \mathcal{O}_{X}$ for some $e \geq 0$ ([T], see also [A2], [OU]). This generalizes what happens in case (1) for curves. On the other hand, works by Sannai-Tanaka [ST], Kitadai-Sumihiro [KS] and Sun [Su] generalize cases (2) and (3) for curves to higher dimension, respectively.

In view of Frobenius splitting, toric varieties are globally $F$-regular (and so $F$-split), and ordinary abelian varieties (e.g., case (2)(i) above) are $F$-split but not globally $F$-regular. In these cases, any direct summand of $F_{*}^{e} \mathcal{O}_{X}$ remains to be a direct summand of $F_{*}^{e^{\prime}} \mathcal{O}_{X}$ for all $e^{\prime} \geq e$. We call such a vector bundle that appears as a direct summand of $F_{*}^{e} \mathcal{O}_{X}$ for some $e \geq 0$ a Frobenius summand on $X$ and ask the following questions.

(a) Does $F_{*}^{e} \mathcal{O}_{X}$ splits into line bundles?

(b) Does there exist only finitely many isomorphism classes of indecomposable Frobenius summands on $X$ ?

If $X$ satisfies the finiteness condition in (b), it is said to be of globally finite $F$-representation type (or GFFRT, for short). It follows from [T] and [ST] that question (a) is affirmative for toric and ordinary abelian varieties, but it is likely that (a) fails for non-toric rational surfaces; cf. Achinger [A2]. On the other hand, the GFFRT property, which breaks down for curves of genus $g \geq 1$, holds true for smooth quadric hypersurfaces of any dimension [A1]. Actually, this follows from the fact that Frobenius summands on a smooth quadric hypersurface are arithmetically Cohen-Macaulay (ACM) bundles and that an ACM bundle on a quadric is either a line bundle or a twisted spinor bundle.

In this paper, we will develop a method to study the structure of the Frobenius push-forward $F_{*}^{e} \mathcal{O}_{X}$ in the case where $X$ is obtained by blowing up the projective plane $\mathbb{P}^{2}$ at $n$ points $P_{1}, \ldots, P_{n}$. Note that such a rational surface $X$ is not toric if $n \geq 4$. As a result, we examine the GFFRT property of $X$ in the simplest non-trivial case, that is, the case where $n=4$. Our main result is the following.

Main Theorem. Let $\pi: X \rightarrow \mathbb{P}^{2}$ be the blowup at four points $P_{1}, P_{2}, P_{3}$, $P_{4} \in \mathbb{P}^{2}$ in general position. Let $H$ be a line in $\mathbb{P}^{2}$ and $E_{i}=\pi^{-1}\left(P_{i}\right)$ the exceptional curve over $P_{i}$. Then any indecomposable Frobenius summand on the surface $X$ coincides with one of the following vector bundles of rank $\leq 3$.

(1) line bundles $\mathcal{O}_{X}, L_{0}=\mathcal{O}_{X}\left(E_{1}+E_{2}+E_{3}+E_{4}-2 \pi^{*} H\right)$ and $L_{i}=$ $\mathcal{O}_{X}\left(E_{i}-\pi^{*} H\right)$ for $i=1,2,3,4$; 
(2) an indecomposable rank 2 bundle $\mathcal{G}$ given by a unique non-trivial extension

$$
0 \rightarrow \mathcal{O}_{X}\left(-\pi^{*} H\right) \rightarrow \mathcal{G} \rightarrow \mathcal{O}_{X}\left(E_{1}+E_{2}+E_{3}+E_{4}-2 \pi^{*} H\right) \rightarrow 0
$$

(3) an indecomposable rank 3 bundle $\mathcal{B}$ given by a non-trivial extension

$$
0 \rightarrow \mathcal{O}_{X}\left(E_{1}-\pi^{*} H\right) \oplus \mathcal{O}_{X}\left(E_{2}-\pi^{*} H\right) \rightarrow \mathcal{B} \rightarrow \mathcal{O}_{X}\left(E_{3}+E_{4}-\pi^{*} H\right) \rightarrow 0 .
$$

Furthermore, for any power $q=p^{e}$ of the characteristic $p$ with $e \geq 1$ one has

$$
F_{*}^{e} \mathcal{O}_{X} \cong \mathcal{O}_{X} \oplus \bigoplus_{i=0}^{4} L_{i}^{\oplus(q-2)} \oplus \mathcal{B} \oplus \mathcal{G}^{\oplus \frac{(q-2)(q-3)}{2}}
$$

We apply the above theorem to show that $F_{*}^{e} \mathcal{O}_{X}$ generates the derived category $D^{b}(X)$ in the sense of [OU]; see Proposition 6.4. In order to prove the theorem, we study the behavior of the Frobenius push-forward and Frobenius summands with respect to the blow-down morphism $\sigma: X \rightarrow Y$ of one of the exceptional curves, say, $E_{4}$. It follows that any Frobenius summand $\mathcal{F}$ on $X$ sits in an exact sequence $0 \rightarrow \sigma^{*} \sigma_{*} \mathcal{F} \rightarrow \mathcal{F} \rightarrow \mathcal{O}_{E_{4}}(-1)^{\oplus n} \rightarrow 0$ with $n \leq \operatorname{rank} \mathcal{F}$ and $\sigma_{*} \mathcal{F}$ is a Frobenius summand on $Y$, which splits into a direct sum of copies of finitely many line bundles since $Y$ is a toric surface. Then it turns out that $\mathcal{F}$ is obtained by successive extension of line bundles either of the form $\sigma^{*} L$ or $\sigma^{*}(L) \otimes \mathcal{O}_{X}\left(E_{4}\right)$, where $L$ is a rank one Frobenius summand on $Y$. Computing the involving extension classes we see that an indecomposable Frobenius summand on $X$ is either one of those listed in (1)-(3) of the theorem. Finally, we can determine the multiplicity of each summand appearing in $F_{*}^{e} \mathcal{O}_{X}$ in terms of the Frobenius splitting method.

The GFFRT property of a blown-up surface of $\mathbb{P}^{2}$ would possibly break down as the number of the blown-up points gets larger than a certain bound. Actually, we can construct an example of a rational surface $X$ obtained by blowing up $\mathbb{P}^{2}$ at more than ten points that is not GFFRT; see Proposition 6.2 .

\section{Preliminaries}

We work over an algebraically closed field $k$ of characteristic $p>0$. It is known that the Frobenius push-forward of any line bundle on a smooth toric variety splits into a direct sum of line bundles [T]; see also [A2], [OU].

1.1. Toric surfaces. We review a few examples for basic toric surfaces.

1.1a. The case $X=\mathbb{P}^{2}$. Let $X$ be the projective plane with homogeneous coordinates $x_{0}, x_{1}, x_{2}$ and let $x=x_{1} / x_{0}, y=x_{2} / x_{0}$. Let $H \subset X$ be the line 
$x_{0}=0$. For $q=p^{e}$ we choose a basis $\left\{x^{i / q} y^{j / q} \mid 0 \leq i, j \leq q-1\right\}$ of $K^{1 / q}$ over the function field $K=k(x, y)$ of $X$. Then we have a decomposition

$$
\begin{aligned}
F_{*}^{e} \mathcal{O}_{X} & =\bigoplus_{0 \leq i, j \leq q-1} L_{i j} x^{i / q} y^{j / q} \\
& \cong \mathcal{O}_{\mathbb{P}^{2}} \oplus \mathcal{O}_{\mathbb{P}^{2}}(-1)^{\oplus \frac{(q-1)(q+4)}{2}} \oplus \mathcal{O}_{\mathbb{P}^{2}}(-2)^{\oplus \frac{(q-1)(q-2)}{2}}
\end{aligned}
$$

with respect to the basis, where

$$
L_{i j}= \begin{cases}\mathcal{O}_{X} & \text { if } i=j=0 \\ \mathcal{O}_{X}(-H) & \text { if } 1 \leq i+j \leq q ; \\ \mathcal{O}_{X}(-2 H) & \text { if } q+1 \leq i+j \leq 2 q-2 .\end{cases}
$$

1.1b. The blowup of $\mathbb{A}^{2}$ at the origin. Let $\pi: X \rightarrow Y=\mathbb{A}^{2}=\operatorname{Spec} k[x, y]$ be the blowup at the origin $o \in \mathbb{A}^{2}$ with the exceptional curve $E=\pi^{-1}(o)$. Then for $q=p^{e}$ we have a decomposition

$$
F_{*}^{e} \mathcal{O}_{X}=\bigoplus_{0 \leq i, j \leq q-1} L_{i j} x^{i / q} y^{j / q} \cong \mathcal{O}_{X}^{\oplus \frac{q(q+1)}{2}} \oplus \mathcal{O}_{X}(E)^{\oplus \frac{q(q-1)}{2}},
$$

where

$$
L_{i j}= \begin{cases}\mathcal{O}_{X} & \text { if } 0 \leq i+j \leq q-1 \\ \mathcal{O}_{X}(E) & \text { if } q \leq i+j \leq 2 q-2 .\end{cases}
$$

In view of the trivial decomposition $F_{*}^{e} \mathcal{O}_{Y} \cong \bigoplus_{0 \leq i, j \leq q-1} \mathcal{O}_{Y} x^{i / q} y^{j / q}$ on $Y=\mathbb{A}^{2}$, we have the following exact sequence

$$
0 \rightarrow \pi^{*} F_{*}^{e} \mathcal{O}_{Y} \rightarrow F_{*}^{e} \mathcal{O}_{X} \rightarrow \mathcal{N} \rightarrow 0,
$$

where $\mathcal{N}=\bigoplus_{q \leq i+j \leq 2 q-2} \mathcal{O}_{E}(E) x^{i / q} y^{j / q} \cong \mathcal{O}_{E}(-1)^{\oplus \frac{q(q-1)}{2}}$.

1.1c. The blowup of $\mathbb{P}^{2}$ at a point. Let $\pi: X \rightarrow \mathbb{P}^{2}$ be the blowup at a torus-invariant point $P \in \mathbb{P}^{2}$ and let $E=\pi^{-1}(P)$. If we choose $P=(1: 0: 0)$, then it follows from (1.1a) and (1.1b) that

$$
\begin{aligned}
F_{*}^{e} \mathcal{O}_{X}= & \bigoplus_{0 \leq i, j \leq q-1} L_{i j} x^{i / q} y^{j / q} \\
\cong & \mathcal{O}_{X} \oplus \mathcal{O}_{X}\left(-\pi^{*} H\right)^{\oplus \frac{q^{2}+q-2}{2}} \oplus \mathcal{O}_{X}\left(E-\pi^{*} H\right)^{\oplus(q-1)} \\
& \oplus \mathcal{O}_{X}\left(E-2 \pi^{*} H\right)^{\oplus \frac{q^{2}-3 q+2}{2}}
\end{aligned}
$$

where

$$
L_{i j}= \begin{cases}\mathcal{O}_{X} & \text { if } i=j=0 \\ \mathcal{O}_{X}\left(-\pi^{*} H\right) & \text { if } 1 \leq i+j \leq q-1 \\ \mathcal{O}_{X}\left(E-\pi^{*} H\right) & \text { if } i+j=q \\ \mathcal{O}_{X}\left(E-2 \pi^{*} H\right) & \text { if } q+1 \leq i+j \leq 2 q-2\end{cases}
$$


Note that the assignment of the summands $\mathcal{O}_{X}\left(-\pi^{*} H\right)$ and $\mathcal{O}_{X}\left(E-\pi^{*} H\right)$ to the pairs $(i, j)$ with $1 \leq i+j \leq q$ depends on the choice of a torus-invariant point $P$. Namely:

(1) if $P=(1: 0: 0)$ then $L_{i j}=\mathcal{O}_{X}\left(E-\pi^{*} H\right)$ for $(i, j)=(1, q-1),(2, q-2)$, $\ldots,(q-1,1)$ as described above, whereas;

(2) if $P=(0: 1: 0)$ then $L_{i j}=\mathcal{O}_{X}\left(E-\pi^{*} H\right)$ for $(i, j)=(0,1), \ldots,(0, q-1)$;

(3) if $P=(0: 0: 1)$ then $L_{i j}=\mathcal{O}_{X}\left(E-\pi^{*} H\right)$ for $(i, j)=(1,0), \ldots,(q-1,0)$.

1.2. The blowup of $\mathbb{P}^{2}$ at more than three points. Let $\pi: X \rightarrow \mathbb{P}^{2}$ be the blowup of the projective plane $\mathbb{P}^{2}=\mathbb{P}_{k}^{2}$ at $n$ points $P_{1}, \ldots, P_{n} \in \mathbb{P}^{2}$ in general position and let $E_{i}=\pi^{-1}\left(P_{i}\right)$ be the exceptional curve over $P_{i}$. We may and will assume that $P_{1}=(1: 0: 0), P_{2}=(0: 1: 0)$ and $P_{3}=(0: 0: 1)$. We factorize $\pi: X \rightarrow \mathbb{P}^{2}$ as $\pi=\sigma \circ \tau$, where $\tau: Y \rightarrow \mathbb{P}^{2}$ is the blowup at $n-1$ points $P_{1}, \ldots, P_{n-1}$ and $\sigma=\sigma_{n}: X \rightarrow Y$ is the blowup at the point $P_{n}$. Then by (1.1) we have an exact sequence

$$
0 \rightarrow \sigma^{*} F_{*}^{e} \mathcal{O}_{Y} \rightarrow F_{*}^{e} \mathcal{O}_{X} \rightarrow \mathcal{O}_{E_{n}}(-1)^{\oplus \frac{q(q-1)}{2}} \rightarrow 0 .
$$

This implies that any direct summand $\mathcal{F}$ of $F_{*}^{e} \mathcal{O}_{X}$ sits in a similar exact sequence

$$
0 \rightarrow \sigma^{*} \mathcal{E} \rightarrow \mathcal{F} \rightarrow \mathcal{N} \rightarrow 0,
$$

where $\mathcal{E}=\pi_{*} \mathcal{F}$ is a direct summand of $F_{*}^{e} \mathcal{O}_{Y}$ and $\mathcal{N}$ is a direct sum of copies of $\mathcal{O}_{E_{n}}(-1)$ 's.

As for line bundle summands of $F_{*}^{e} \mathcal{O}_{X}$, we easily see the following: If a line bundle $L$ is a direct summand of $F_{*}^{e} \mathcal{O}_{X}$, then $L^{\prime}=\sigma_{*} L$ is also a direct summand on $F_{*}^{e} \mathcal{O}_{Y}$, and $L$ sits in an exact sequence

$$
0 \rightarrow \sigma^{*} L^{\prime} \rightarrow L \rightarrow \mathcal{N} \rightarrow 0
$$

with $\mathcal{N}=0$ or $\mathcal{O}_{E_{n}}(-1)$. Hence $L \cong \sigma^{*} L^{\prime}$ or $\sigma^{*}\left(L^{\prime}\right) \otimes \mathcal{O}_{X}\left(E_{n}\right)$.

We want to look out for all indecomposable Frobenius summands on $X$, that is, indecomposable vector bundles appearing as a direct summand of $F_{*}^{e} \mathcal{O}_{X}$ for some $e \geq 0$. This problem is closely related to $F$-splitting property: $X$ is said to be $F$-split if the Frobenius map $F: \mathcal{O}_{X} \rightarrow F_{*} \mathcal{O}_{X}$ splits as an $\mathcal{O}_{X^{-}}$ module homomorphism, or equivalently, $F_{*}^{e} \mathcal{O}_{X}$ has a free direct summand $\mathcal{O}_{X}$ for all $e \geq 0 .^{1}$

The following are known for the $F$-splitting property of smooth del Pezzo surfaces.

Proposition 1.1 (Cf. [H, Example 5.5]). Assume that $n \leq 8$ under the notation of Section 1.2 above. Then $X$ is F-split except for the following three cases.

(1) $n=6$ and $p=2$;

\footnotetext{
${ }^{1}$ When $X$ is projective over $k$, the maximal rank of a free Frobenius summand is at most 1 , because $H^{0}\left(X, F_{*}^{e} \mathcal{O}_{X}\right)=k$.
} 
(2) $n=7$ and $p=2$ or 3 ;

(3) $n=8$ and $p=2,3$ or 5 .

Our main target in this paper is the case $n=4$, where $X$ itself is a non-toric $F$-split surface but the surface $Y$ downstairs, being the blowup of $\mathbb{P}^{2}$ at three points $P_{1}, P_{2}, P_{3}$, is toric. Summing up the results in $(1.1 \mathrm{c})$, we obtain the following lemma.

Lemma 1.2. Let $\tau: Y \rightarrow \mathbb{P}^{2}$ be the blowup at the three points $P_{1}, P_{2}, P_{3}$ in general position. Then $F_{*}^{e} \mathcal{O}_{Y}$ is isomorphic to

$$
\begin{gathered}
\mathcal{O}_{Y} \oplus \mathcal{O}_{Y}\left(-\tau^{*} H\right)^{\oplus \frac{(q-1)(q-2)}{2}} \oplus \bigoplus_{i=1}^{3} \mathcal{O}_{Y}\left(E_{i}-\tau^{*} H\right)^{\oplus(q-1)} \\
\oplus \mathcal{O}_{Y}\left(E^{\prime}-2 \tau^{*} H\right)^{\oplus \frac{(q-1)(q-2)}{2}}
\end{gathered}
$$

where $q=p^{e}$ and $E^{\prime}=E_{1}+E_{2}+E_{3}$.

LEMMA 1.3. Under the notation as in Lemma 1.2 we also have

$$
\begin{aligned}
F_{*}^{e} \mathcal{O}_{Y}\left(-\tau^{*} H\right) & \cong \mathcal{O}_{Y}\left(-\tau^{*} H\right)^{\oplus \frac{q(q+1)}{2}} \oplus \mathcal{O}_{Y}\left(E^{\prime}-2 \tau^{*} H\right)^{\oplus \frac{q(q-1)}{2}}, \\
F_{*}^{e} \mathcal{O}_{Y}\left(E^{\prime}-2 \tau^{*} H\right) & \cong \mathcal{O}_{Y}\left(-\tau^{*} H\right)^{\oplus \frac{q(q-1)}{2}} \oplus \mathcal{O}_{Y}\left(E^{\prime}-2 \tau^{*} H\right)^{\oplus \frac{q(q+1)}{2}}
\end{aligned}
$$

and

$$
\begin{aligned}
& F_{*}^{e} \mathcal{O}_{Y}\left(E_{i}-\tau^{*} H\right) \\
& \quad \cong \mathcal{O}_{Y}\left(-\tau^{*} H\right)^{\oplus \frac{q(q-1)}{2}} \oplus \mathcal{O}_{Y}\left(E_{i}-\tau^{*} H\right)^{\oplus q} \oplus \mathcal{O}_{Y}\left(E^{\prime}-2 \tau^{*} H\right)^{\oplus \frac{q(q-1)}{2}}
\end{aligned}
$$

for $i=1,2,3$.

Proof. The proof is similar to Lemma 1.2 and left to the reader.

Using the above lemmas, we can show that any Frobenius summand on a four-point blowup of $\mathbb{P}^{2}$ is obtained by successive extensions of line bundles. To analyze the extensions we need to know the dimension of the extension group $\operatorname{Ext}^{1}(L, M)$ for line bundles $L, M$. Since $\operatorname{Ext}^{1}(L, M) \cong$ $H^{1}\left(X, L^{-1} \otimes M\right)$, its dimension is computed with Riemann-Roch. We collect a few such computations that will be used later; see also Lemma 4.3.

LEMMA 1.4. Assume $n=4$ under the notation of Section 1.2 above, let $E=E_{1}+E_{2}+E_{3}+E_{4}$ and let $i, j, l \in\{1,2,3,4\}$ be any distinct indexes. Then the first cohomology groups

$$
\begin{array}{ll}
H^{1}\left(X, \mathcal{O}_{X}\left(\pi^{*} H-E\right)\right), & H^{1}\left(X, \mathcal{O}_{X}\left(E-2 E_{i}-\pi^{*} H\right)\right), \\
H^{1}\left(X, \mathcal{O}_{X}\left(E_{i}-E_{j}-E_{l}\right)\right) &
\end{array}
$$

are one-dimensional $k$-vector spaces. On the other hand, $H^{1}\left(X, \mathcal{O}_{X}(D)\right)=0$ holds for $D= \pm E_{i}, E_{i}-E_{j}, 2 \pi^{*} H-E$ and $\pi^{*} H-E^{\prime \prime}$ with $0 \leq E^{\prime \prime} \leq E-E_{i}$. 
Proof. Let $h^{i}=h^{i}\left(\mathcal{O}_{X}\left(\pi^{*} H-E\right)\right)$ for $i=0,1,2$. Then

$$
\chi\left(\mathcal{O}_{X}\left(\pi^{*} H-E\right)\right)=h^{0}-h^{1}+h^{2}=\frac{1}{2}\left(\pi^{*} H-E\right)\left(\pi^{*} H-E-K_{X}\right)+1=-1
$$

by the Riemann-Roch formula. Here we used the fact that $K_{X} \sim E-$ $3 \pi^{*} H$. On the other hand, $h^{0}=0$ since $P_{1}, P_{2}, P_{3}, P_{4}$ are not collinear, and $h^{2}=h^{0}\left(\mathcal{O}_{X}\left(K_{X}-\pi^{*} H+E\right)\right)=0$ by the Serre duality. Thus $h^{1}=$ $h^{1}\left(\mathcal{O}_{X}\left(\pi^{*} H-E\right)\right)=1$, as required.

The dimensions of the other first cohomology groups are computed similarly. We just discuss the vanishing of $H^{1}\left(X, \mathcal{O}_{X}\left(\pi^{*} H-E^{\prime \prime}\right)\right)$ from them. Let $E^{\prime \prime}$ be the sum of $r$ distinct $(-1)$-curves with $0 \leq r \leq 3$. Then $\chi\left(\mathcal{O}_{X}\left(\pi^{*} H-E^{\prime \prime}\right)\right)=3-r$ by Riemann-Roch and $h^{2}\left(\mathcal{O}_{X}\left(\pi^{*} H-E^{\prime \prime}\right)\right)=0$ by the Serre duality. It also follows that $h^{0}\left(\mathcal{O}_{X}\left(\pi^{*} H-E^{\prime \prime}\right)\right)=3-r$ from the condition of general position that no three of $P_{1}, P_{2}, P_{3}, P_{4}$ are collinear. Thus, $h^{1}\left(\mathcal{O}_{X}\left(\pi^{*} H-E^{\prime \prime}\right)\right)=0$, as required.

\section{The behavior of Frobenius summands under point-blowups}

Our strategy to study Frobenius summands on a four-point blowup $X$ of $\mathbb{P}^{2}$ is to factorize $\pi: X \rightarrow \mathbb{P}^{2}$ as $\pi=\sigma \circ \tau$ as in Section 1.2 and look at what happens for the blowup $\sigma: X \rightarrow Y$ at $P_{4}$. For this purpose, we will work under the following setup in this section. Note that Frobenius summands on the blowup of $\mathbb{P}^{2}$ at four points satisfy conditions (a), (b) and (c) below; see Section 1 and especially (1.2).

2.1. Setup. Let $\sigma: X \rightarrow Y$ be the blowup at a point $P$ on a smooth projective surface $Y$ with exceptional curve $E=\sigma^{-1}(P)$. Let $\mathcal{F}$ be a vector bundle of rank $r$ on $X$ satisfying the following conditions:

(a) $\sigma_{*} \mathcal{F} \cong L_{1} \oplus \cdots \oplus L_{r}$ for line bundles $L_{1}, \ldots, L_{r}$ on $Y$.

(b) $\mathcal{F}$ sits in an exact sequence

$$
0 \rightarrow \sigma^{*} \sigma_{*} \mathcal{F} \rightarrow \mathcal{F} \rightarrow \mathcal{O}_{E}(-1)^{\oplus n} \rightarrow 0
$$

where $n$ is an integer with $0 \leq n \leq r$.

(c) $H^{i}(X, \mathcal{F})=0$ for $i>0$.

Under condition (b), condition (c) holds if and only if $H^{i}\left(X, \sigma^{*} \sigma_{*} \mathcal{F}\right)=0$ for $i>0$, or equivalently if $H^{i}\left(Y, L_{j}\right)=H^{i}\left(X, \sigma^{*} L_{j}\right)=0$ for $i>0$ and $1 \leq j \leq r$. Note that this vanishing holds if $L_{j}$ 's are Frobenius summands on a rational surface $Y$, since $H^{i}\left(Y, L_{j}\right) \subseteq H^{i}\left(Y, F_{*}^{e} \mathcal{O}_{Y}\right)=H^{i}\left(Y, \mathcal{O}_{Y}\right)$.

Let $\mathcal{F}$ be a vector bundle of rank $r$ on $X$ satisfying conditions (a)-(c) as above. Let $\mathcal{E}=\sigma_{*} \mathcal{F} \cong L_{1} \oplus \cdots \oplus L_{r}, \mathcal{E}^{\prime}=L_{1} \oplus \cdots \oplus L_{r-1}$ and let $\mathcal{F}^{\prime}$ be the saturation of $\sigma^{*} \mathcal{E}^{\prime}$ in $\mathcal{F}$, that is, $\mathcal{F}^{\prime}$ is the kernel of the natural map $\mathcal{F} \rightarrow\left(\mathcal{F} / \sigma^{*} \mathcal{E}^{\prime}\right) / \operatorname{tors}\left(\mathcal{F} / \sigma^{*} \mathcal{E}^{\prime}\right)$. Then $\mathcal{F}^{\prime}$, which satisfies Serre's condition $\left(\mathrm{S}_{2}\right)$ 
on a smooth surface, is a vector bundle of rank $r-1$, since $\mathcal{F} / \mathcal{F}^{\prime}$ is a torsionfree $\mathcal{O}_{X}$-module of rank 1 . We have the following commutative diagram

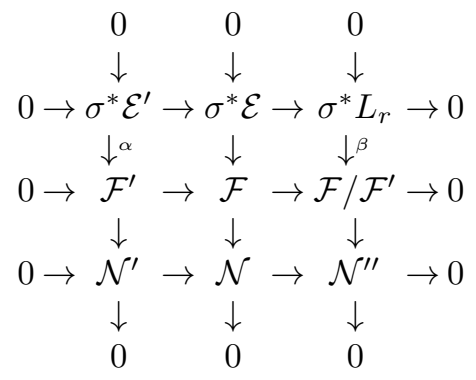

with exact rows and columns, where $\mathcal{N}=\mathcal{O}_{E}(-1)^{\oplus n}$, and $\mathcal{N}^{\prime}$ and $\mathcal{N}^{\prime \prime}$ are the cokernels of the maps $\alpha$ and $\beta$, respectively. Since $\mathcal{F} / \mathcal{F}^{\prime}$ is isomorphic to $\sigma^{*} L_{r}$ outside $E$, one has $\left(\mathcal{F} / \mathcal{F}^{\prime}\right) \otimes \sigma^{*} L_{r}^{-1} \cong \mathcal{I}_{Z} \otimes \mathcal{O}_{X}(m E)$ for an integer $m \geq 0$, where $Z$ is a 0 -dimensional closed subscheme of $X$ supported in $E$. Actually, we must have $m=0$ or 1 since $\mathcal{N}^{\prime \prime}=\operatorname{Coker}(\beta)$ has an $\mathcal{O}_{E}$-module structure induced from the surjection $\mathcal{N} \rightarrow \mathcal{N}^{\prime \prime}$, and $m=1$ if $Z$ is non-empty.

CLAim 2.1. $\mathcal{N}^{\prime} \cong \mathcal{O}_{E}(-1)^{\oplus n-m}, \mathcal{N}^{\prime \prime} \cong \mathcal{O}_{E}(-1)^{\oplus m}$ and $Z=\emptyset$.

Proof. Let $M=\sigma^{*}\left(L_{r}\right) \otimes \mathcal{O}_{X}(m E)$ and look at the exact sequence

$$
0 \rightarrow \mathcal{F}^{\prime} \rightarrow \mathcal{F} \rightarrow \mathcal{I}_{Z} \otimes M \rightarrow 0
$$

Then we have

$$
\chi(\mathcal{F})=\chi\left(\mathcal{F}^{\prime}\right)+\chi\left(\mathcal{I}_{Z} \otimes M\right)=\chi\left(\mathcal{F}^{\prime}\right)+\chi(M)-\operatorname{deg} Z .
$$

The Euler characteristic on the left-hand side is computed as

$$
\chi(\mathcal{F})=h^{0}(\mathcal{F})=h^{0}\left(\sigma^{*} \mathcal{E}\right)=h^{0}\left(\sigma^{*} \mathcal{E}^{\prime}\right)+h^{0}\left(\sigma^{*} L_{r}\right),
$$

where the equalities follow from the vanishing (c), the exact sequence in (b) together with $h^{0}(\mathcal{N})=0$, and the splitting $\sigma^{*} \mathcal{E} \cong \sigma^{*} \mathcal{E}^{\prime} \oplus \sigma^{*} L_{r}$, respectively. On the other hand, it follows that $\chi(M)=\chi\left(\sigma^{*} L_{r}\right)+m \chi\left(\mathcal{O}_{E}(-1)\right)=h^{0}\left(\sigma^{*} L_{r}\right)$ from the exact sequence $0 \rightarrow \sigma^{*} L_{r} \rightarrow M \rightarrow \mathcal{O}_{E}(-1)^{\oplus m} \rightarrow 0$ with $m=0,1$. We also have that $H^{i}\left(X, \sigma^{*} \mathcal{E}^{\prime}\right)=0$ for $i>0$, and all but the first cohomology groups of $\mathcal{N}^{\prime}$ vanish because of the monomorphism $\mathcal{N}^{\prime} \hookrightarrow \mathcal{N}=\mathcal{O}_{E}(-1)^{\oplus n}$. Looking at the exact sequence $0 \rightarrow \sigma^{*} \mathcal{E}^{\prime} \rightarrow \mathcal{F}^{\prime} \rightarrow \mathcal{N}^{\prime} \rightarrow 0$, we know that $H^{0}\left(X, \sigma^{*} \mathcal{E}^{\prime}\right) \cong H^{0}\left(X, \mathcal{F}^{\prime}\right), H^{2}\left(X, \mathcal{F}^{\prime}\right)=0$ and that $\chi\left(\mathcal{F}^{\prime}\right)=h^{0}\left(\mathcal{F}^{\prime}\right)-h^{1}\left(\mathcal{F}^{\prime}\right)=$ $h^{0}\left(\sigma^{*} \mathcal{E}^{\prime}\right)-h^{1}\left(\mathcal{F}^{\prime}\right)$. Summing up, we have $h^{1}\left(\mathcal{F}^{\prime}\right)+\operatorname{deg} Z=0$, so that $h^{1}\left(\mathcal{F}^{\prime}\right)=$ $\operatorname{deg} Z=0$. Thus, $Z=\emptyset$ and $\mathcal{N}^{\prime \prime} \cong \mathcal{O}_{E}(-1)^{\oplus m}$. It then follows that the exact sequence $0 \rightarrow \mathcal{N}^{\prime} \rightarrow \mathcal{N} \rightarrow \mathcal{N}^{\prime \prime} \rightarrow 0$ splits and $\mathcal{N}^{\prime} \cong \mathcal{O}_{E}(-1)^{\oplus n-m}$.

Thus, we are led to the following. 
LEMMA 2.1. Let $\mathcal{F}$ be a vector bundle of rank $r$ on $X$ satisfying conditions (a), (b) and (c) as in Section 2.1. Then there exists a filtration $0=\mathcal{F}_{0} \subset \mathcal{F}_{1} \subset$ $\cdots \subset \mathcal{F}_{r}=\mathcal{F}$ of subbundles $\mathcal{F}_{i}$ with line bundle quotients $\mathcal{F}_{i} / \mathcal{F}_{i-1}$ such that the following hold for $1 \leq i \leq r$.

(1) $\mathcal{F}_{i}$ satisfies conditions (a), (b) and (c) with $\sigma_{*} \mathcal{F}_{i} \cong L_{1} \oplus \cdots \oplus L_{i}$.

(2) $\mathcal{F}_{i} / \mathcal{F}_{i-1} \cong \sigma^{*} L_{i}$ or $\sigma^{*}\left(L_{i}\right) \otimes \mathcal{O}_{X}(E)$. In particular, if $\mathcal{F}_{i}$ is indecomposable, then $\mathcal{F}_{i} / \mathcal{F}_{i-1} \cong \sigma^{*}\left(L_{r}\right) \otimes \mathcal{O}_{X}(E)$.

Proof. For each $i$ let $\mathcal{F}_{i}$ be the saturation of $\sigma^{*}\left(L_{1} \oplus \cdots \oplus L_{i}\right)$ in $\mathcal{F}$. Then $\mathcal{F}_{i}$ is a vector bundle of rank $i$ with $\sigma_{*} \mathcal{F}_{i} \cong L_{1} \oplus \cdots \oplus L_{i}$. Moreover, it follows from the argument above that assertions (1) for $i=r-1$ and (2) for $i=r$ hold. Since $\mathcal{F}_{r-1}$ satisfies conditions (a), (b) and (c), we obtain the whole statement (except for the case when $\mathcal{F}_{i}$ is indecomposable) by descending induction on $i$.

Finally, suppose that $\mathcal{F}_{i}$ is indecomposable and $\mathcal{F}_{i} / \mathcal{F}_{i-1} \cong \sigma^{*} L_{i}$. Then the splitting map $\sigma^{*} L_{i} \rightarrow \sigma^{*} \sigma_{*} \mathcal{F}_{i}$ composed with the inclusion map $\sigma^{*} \sigma_{*} \mathcal{F}_{i} \rightarrow \mathcal{F}_{i}$ gives a splitting of the map $\mathcal{F}_{i} \rightarrow \mathcal{F}_{i} / \mathcal{F}_{i-1}$, which contradicts to the indecomposability of $\mathcal{F}_{i}$.

Corollary 2.2. Let $\mathcal{F}$ be a vector bundle of rank $r$ on $X$ satisfying conditions (a), (b) and (c). For any subset $I=\left\{i_{1}, \ldots, i_{s}\right\} \subset\{1, \ldots, r\}$ with $J=\{1, \ldots, r\} \backslash I$, let $\mathcal{F}_{I}$ be the saturation of $\sigma^{*}\left(L_{i_{1}} \oplus \cdots \oplus L_{i_{s}}\right)$ in $\mathcal{F}$ and let $\overline{\mathcal{F}}_{J}=\mathcal{F} / \mathcal{F}_{I}$. Then $\mathcal{F}_{I}$ and $\overline{\mathcal{F}}_{J}$ are vector bundles of rank $s$ and $r-s$, respectively, satisfying conditions (a), (b) and (c) with $\sigma_{*} \mathcal{F}_{I} \cong \bigoplus_{i \in I} L_{i}$ and $\sigma_{*} \overline{\mathcal{F}}_{J} \cong \bigoplus_{j \in J} L_{j}$.

Proof. We will prove our assertion by descending induction on $s=\sharp I$. The case $s=r$ is trivial. Suppose our assertion holds for $s$ with $0<s \leq r$ and consider the following commutative diagram with exact rows and columns. ${ }^{2}$

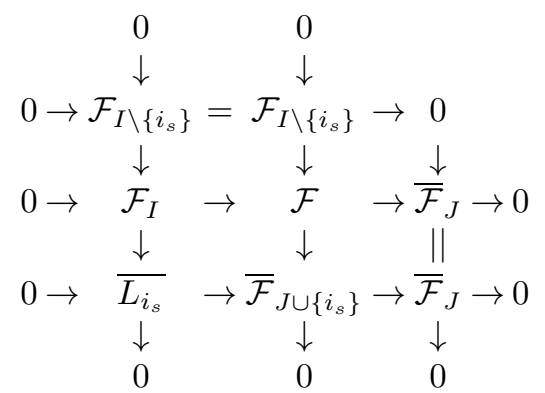

Since $\mathcal{F}_{I}$ and $\overline{\mathcal{F}}_{J}$ satisfy the required property by induction hypothesis, it follows from Lemma 2.1 that $\mathcal{F}_{I \backslash\left\{i_{s}\right\}}$ satisfies conditions (a)-(c) and that

\footnotetext{
2 Note that the saturation of $\sigma^{*}\left(L_{i_{1}} \oplus \cdots \oplus L_{i_{s-1}}\right)$ in $\mathcal{F}_{I}$ coincides with the saturation of $\mathcal{F}_{I \backslash\left\{i_{s}\right\}}$ in $\mathcal{F}$.
} 
$\overline{L_{i_{s}}} \cong \sigma^{*} L_{i_{s}}$ or $\sigma^{*}\left(L_{i_{s}}\right) \otimes \mathcal{O}_{X}(E)$. Looking at the bottom exact sequence we see that $\overline{\mathcal{F}}_{J \cup\left\{i_{s}\right\}}$ also satisfies conditions (a)-(c).

As for indecomposable bundles of ranks 2 and 3, we have the following corollary.

COROLlary 2.3. Under the notation of Section 2.1, assume that $\mathcal{F}$ is indecomposable of rank 2 such that $\sigma_{*} \mathcal{F} \cong L \oplus M$ for line bundles $L$ and $M$ on $Y$. Then we have an exact sequence

$$
0 \rightarrow \sigma^{*} L \rightarrow \mathcal{F} \rightarrow \sigma^{*}(M) \otimes \mathcal{O}_{X}(E) \rightarrow 0
$$

given by a non-zero extension class $\varepsilon \in \operatorname{Ext}^{1}\left(\sigma^{*}(M) \otimes \mathcal{O}_{X}(E), \sigma^{*} L\right)$ sitting in $\operatorname{Ker}\left(\operatorname{Ext}^{1}\left(\sigma^{*}(M) \otimes \mathcal{O}_{X}(E), \sigma^{*} L\right) \rightarrow \operatorname{Ext}^{1}\left(\sigma^{*} M, \sigma^{*} L\right) \cong \operatorname{Ext}^{1}(M, L)\right) \cong k$.

Proof. By Lemma 2.1, we have an exact sequence

$$
0 \rightarrow \widetilde{L} \rightarrow \mathcal{F} \rightarrow \sigma^{*}(M) \otimes \mathcal{O}_{X}(E) \rightarrow 0
$$

with $\widetilde{L} \cong \sigma^{*} L$ or $\sigma^{*}(L) \otimes \mathcal{O}_{X}(E)$. Suppose that $\widetilde{L} \cong \sigma^{*}(L) \otimes \mathcal{O}_{X}(E)$. Then the inclusion map $\mathcal{F} \rightarrow \sigma^{*} \sigma_{*}(\mathcal{F}) \otimes \mathcal{O}_{X}(E)$ composed with the map $\sigma^{*} \sigma_{*}(\mathcal{F}) \otimes$ $\mathcal{O}_{X}(E) \rightarrow \sigma^{*}(L) \otimes \mathcal{O}_{X}(E)$, which is induced by the splitting map $\sigma_{*} \mathcal{F} \rightarrow L$, gives a splitting of the map $\widetilde{L} \rightarrow \mathcal{F}$. This absurdity implies that $\widetilde{L} \cong \sigma^{*} L$.

Next, we will show that the kernel of the natural map $\operatorname{Ext}^{1}(\bar{M}, \widetilde{L}) \stackrel{\rho}{\rightarrow}$ $\operatorname{Ext}^{1}(M, L)$ is a vector space of dimension $\leq 1$. To this end, we consider the exact sequence

$$
0 \rightarrow \sigma^{*} M \rightarrow \sigma^{*}(M) \otimes \mathcal{O}_{X}(E) \rightarrow \mathcal{O}_{E}(-1) \rightarrow 0
$$

and apply the functor $\operatorname{Ext}^{\bullet}\left(-, \sigma^{*} L\right)$ to obtain a long exact sequence

$$
\operatorname{Ext}^{1}\left(\mathcal{O}_{E}(-1), \sigma^{*} L\right) \rightarrow \operatorname{Ext}^{1}\left(\sigma^{*}(M) \otimes \mathcal{O}_{X}(E), \sigma^{*} L\right) \stackrel{\rho}{\rightarrow} \operatorname{Ext}^{1}\left(\sigma^{*} M, \sigma^{*} L\right) \rightarrow 0 .
$$

It is sufficient to show that $\operatorname{Ext}^{1}\left(\mathcal{O}_{E}(-1), \sigma^{*} L\right)$ is 1-dimensional. But this follows because $\operatorname{Ext}^{1}\left(\mathcal{O}_{E}(-1), \sigma^{*} L\right) \cong H^{0}\left(X, \mathcal{E} x t^{1}\left(\mathcal{O}_{E}(-1), \sigma^{*} L\right)\right)$ by the Leray spectral sequence and

$$
\begin{aligned}
\mathcal{E} x t^{1}\left(\mathcal{O}_{E}(-1), \sigma^{*} L\right) & \cong \mathcal{E} x t^{1}\left(\mathcal{O}_{E}, \omega_{X}\right) \otimes \omega_{X}(E)^{-1} \otimes \sigma^{*} L \\
& \cong \omega_{E} \otimes \omega_{X}(E)^{-1} \otimes \sigma^{*} L \\
& \cong \mathcal{O}_{E} .
\end{aligned}
$$

Corollary 2.4. Under the notation of Section 2.1, assume that $\mathcal{F}$ is indecomposable of rank 3 with $\sigma_{*} \mathcal{F} \cong L_{1} \oplus L_{2} \oplus L_{3}$. Then we have a nontrivial extension

$$
0 \rightarrow \widetilde{L_{1} \oplus L_{2}} \rightarrow \mathcal{F} \rightarrow \sigma^{*}\left(L_{3}\right) \otimes \mathcal{O}_{X}(E) \rightarrow 0,
$$

and either one of the following two cases occurs.

(1) $\widetilde{L_{1} \oplus L_{2}}=\sigma^{*}\left(L_{1}\right) \oplus \sigma^{*}\left(L_{2}\right)$ with $L_{1} ¥ L_{2}$.

(2) $\widetilde{L_{1} \oplus L_{2}}$ is indecomposable and $\operatorname{det}\left(\widetilde{L_{1} \oplus L_{2}}\right) \cong \sigma^{*}\left(L_{1} \otimes L_{2}\right) \otimes \mathcal{O}_{X}(E)$. 
Proof. The proof goes similarly as in Corollary 2.3. We just show the reason why $L_{1} ¥ L_{2}$ in (1), as well as the indecomposability in (2).

Suppose that $L_{1} \cong L_{2}$ in case (1) and let $\mathcal{F}$ be given by an extension class $\varepsilon=\left(\varepsilon_{1}, \varepsilon_{2}\right)$, where

$$
\varepsilon_{i} \in K_{i}=\operatorname{Ker}\left(\operatorname{Ext}^{1}\left(\sigma^{*}\left(L_{3}\right) \otimes \mathcal{O}_{X}(E), \sigma^{*} L_{i}\right) \rightarrow \operatorname{Ext}^{1}\left(L_{3}, L_{i}\right)\right)
$$

for $i=1,2$. Then $K_{1} \cong K_{2}$ is a 1 -dimensional vector space as we have seen in the proof of Lemma 2.3. It follows that an automorphism of $\sigma^{*} L_{1} \oplus \sigma^{*} L_{2}$ transforms $\varepsilon$ to an extension class of the form $\left(\varepsilon^{\prime}, 0\right)$. Then $\mathcal{F}$ splits as $\mathcal{F} \cong$ $\mathcal{F}^{\prime} \oplus \sigma^{*} L_{2}$, where $\mathcal{F}^{\prime}$ is a rank 2 bundle given by an extension class $\varepsilon^{\prime}$.

Suppose now that $\widetilde{L_{1} \oplus L_{2}}$ is a decomposable bundle with determinant $\sigma^{*}\left(L_{1} \otimes L_{2}\right) \otimes \mathcal{O}_{X}(E)$. It then follows from $\sigma_{*}\left(\widetilde{L_{1} \oplus L_{2}}\right) \cong L_{1} \oplus L_{2}$ that $\widetilde{L_{1} \oplus L_{2}} \cong\left(\sigma^{*}\left(L_{1}\right) \otimes \mathcal{O}_{X}\left(E_{4}\right)\right) \oplus \sigma^{*} L_{2}$ or $\sigma^{*} L_{1} \oplus\left(\sigma^{*}\left(L_{2}\right) \otimes \mathcal{O}_{X}\left(E_{4}\right)\right)$. In each case $\mathcal{F}$ is given by an extension class of the form $\left(\varepsilon_{1}, 0\right)$ or $\left(0, \varepsilon_{2}\right)$, respectively. This contradicts the indecomposability of $\mathcal{F}$.

\section{Frobenius summands of rank 2 and 3 on a four-point blowup of $\mathbb{P}^{2}$}

We will apply Corollaries 2.3 and 2.4 to the case $n=4$ in the notation of Section 1.2. Namely, $\pi: X \rightarrow \mathbb{P}^{2}$ is the blowup of $\mathbb{P}^{2}$ at four points $P_{1}, P_{2}, P_{3}, P_{4}$ in general position. We factorize $\pi$ as

$$
\pi: X \stackrel{\sigma}{\rightarrow} Y \stackrel{\tau}{\rightarrow} \mathbb{P}^{2}
$$

where $\tau$ is the blowup at the 3 points $P_{1}, P_{2}, P_{3}$.

3.1. Rank 2 Frobenius summands on a four-point blowup. We will find out indecomposable rank 2 Frobenius summands on $X$ with a non-trivial extension

$$
0 \rightarrow \sigma^{*} L \rightarrow \mathcal{F} \rightarrow \sigma^{*}(M) \otimes \mathcal{O}_{X}\left(E_{4}\right) \rightarrow 0
$$

where $L$ and $M$ are non-trivial line bundles that are Frobenius summands on $Y$. In this case, Lemma 1.2 tells us that $L$ and $M$ are isomorphic to either $\mathcal{O}_{Y}\left(-\tau^{*} H\right), \mathcal{O}_{Y}\left(E_{i}-\tau^{*} H\right)$ with $i=1,2,3$, or $\mathcal{O}_{Y}\left(E^{\prime}-2 \tau^{*} H\right)$, where $H$ is a line on $\mathbb{P}^{2}$ and $E^{\prime}=E_{1}+E_{2}+E_{3}$.

We give two candidates for rank 2 Frobenius summands in Examples 3.1 and 3.3, from which the latter one will be ruled out.

ExAmPLE 3.1. Let $L=\mathcal{O}_{Y}\left(-\tau^{*} H\right)$ and $M=\mathcal{O}_{Y}\left(E^{\prime}-2 \tau^{*} H\right)$. Then

$$
\operatorname{dim} \operatorname{Ext}^{1}\left(\sigma^{*}(M) \otimes \mathcal{O}_{X}\left(E_{4}\right), \sigma^{*} L\right)=h^{1}\left(\mathcal{O}_{X}\left(\pi^{*} H-E\right)\right)=1
$$

by Lemma 1.4, where $E=E_{1}+E_{2}+E_{3}+E_{4}=\sigma^{*} E^{\prime}+E_{4}$. Hence we have a unique non-trivial extension as (3.1) above that gives a vector bundle $\mathcal{G}$ of rank 2 ,

$$
0 \rightarrow \mathcal{O}_{X}\left(-\pi^{*} H\right) \rightarrow \mathcal{G} \rightarrow \mathcal{O}_{X}\left(E-2 \pi^{*} H\right) \rightarrow 0
$$


Since $\operatorname{Ext}^{1}(M, L) \cong H^{0}\left(Y, \mathcal{O}_{Y}\left(\tau^{*} H-E^{\prime}\right)\right) \cong H^{1}\left(X, \mathcal{O}_{X}\left(\pi^{*} H-E+E_{4}\right)\right)=0$ by Lemma 1.4, the natural map between the extension groups

$$
\operatorname{Ext}^{1}\left(\sigma^{*}(M) \otimes \mathcal{O}_{X}\left(E_{4}\right), \sigma^{*} L\right) \rightarrow \operatorname{Ext}^{1}(M, L)=0
$$

is the zero map, and we do have $\sigma_{*} \mathcal{G} \cong L \oplus M$. To see that $\mathcal{G}$ is indecomposable, let $\sigma_{i}: X \rightarrow Y_{i}$ be the blow-down of $E_{i}$ for $i=1,2,3,4$. Then by symmetry we have

$$
\left(\sigma_{i}\right)_{*} \mathcal{G} \cong \mathcal{O}_{Y_{i}}\left(-\pi_{i}^{*} H\right) \oplus \mathcal{O}_{Y_{i}}\left(E^{(i)}-2 \pi_{i}^{*} H\right),
$$

where $E^{(i)}=\left(\sigma_{i}\right)_{*} E$ is the sum of three exceptional curves of $\pi_{i}: Y_{i} \rightarrow \mathbb{P}^{2}$. Suppose that $\mathcal{G}$ is decomposable. Then we can write $\mathcal{G} \cong \mathcal{O}_{X}\left(D_{1}-\pi^{*} H\right) \oplus$ $\mathcal{O}_{X}\left(D_{2}-2 \pi^{*} H\right)$, where $D_{1}$ and $D_{2}$ are effective $\pi$-exceptional divisors with $D_{1}+D_{2}=E$. Pushing it out by $\sigma_{i}$ and comparing with the splitting above, we see that $D_{2} \geq E^{(i)}$ for all $i$. This implies that $D_{1}=0$ and $D_{2}=E$, so that $\mathcal{G} \cong \mathcal{O}_{X}\left(-\pi^{*} H\right) \oplus \mathcal{O}_{X}\left(E-2 \pi^{*} H\right)$. However, this contradicts to the nontriviality of the extension (3.2).

Since $\mathcal{G}$ is indecomposable with $\sigma_{*} \mathcal{G} \cong \mathcal{O}_{Y}\left(-\tau^{*} H\right) \oplus \mathcal{O}_{Y}\left(E^{\prime}-2 \tau^{*} H\right)$, we can replace $L$ and $M$ with each other to see that it sits in another unique non-trivial extension; cf. Lemma 1.4:

$$
0 \rightarrow \mathcal{O}_{X}\left(E^{\prime}-2 \pi^{*} H\right) \rightarrow \mathcal{G} \rightarrow \mathcal{O}_{X}\left(E_{4}-\pi^{*} H\right) \rightarrow 0 .
$$

Remark 3.2. We need the condition that the blown-up points $P_{1}, P_{2}, P_{3}, P_{4}$ are in general position for the symmetry used in Example 3.1, specifically for the vanishing $H^{1}\left(X, \mathcal{O}_{X}\left(\pi^{*} H-E+E_{i}\right)\right)=0$ for $i=1,2,3$, 4; cf. Lemma 1.4.

The condition of general position allows us to assume that $P_{1}=(1: 0: 0)$, $P_{2}=(0: 1: 0), P_{3}=(0: 0: 1)$ and $P_{4}=(1: 1: 1)$. It then turns out that exact sequence (3.3) is the pullback of (3.2) by an automorphism on $X$. Indeed, the canonical Cremona transformation $\left(x_{0}: x_{1}: x_{2}\right) \mapsto\left(x_{1} x_{2}: x_{2} x_{0}: x_{0} x_{1}\right)$ on $\mathbb{P}^{2}$ lifts to an involution $\varphi$ on $X$, which transforms $\pi^{*}(2 H)-E^{\prime}$ to $\pi^{*} H$ and fixes $E_{4}$. Hence, (3.3) is the pullback of $(3.2)$ by $\varphi$, so that $\varphi^{*} \mathcal{G} \cong \mathcal{G}$.

On the other hand, for $1 \leq i<j \leq 4$ one has a unique automorphism on $\mathbb{P}^{2}$ (i.e., an element of $\operatorname{PGL}(2, k))$ that permutes $P_{i}$ and $P_{j}$ and fixes the other two blown up points. It is easy to see that it lifts to an involution $\psi_{i j}$ of $X$ such that $\psi_{i j}^{*} \mathcal{G} \cong \mathcal{G}$.

EXAMPLE 3.3. Let $L=\mathcal{O}_{Y}\left(E_{1}-\tau^{*} H\right)$ and $M=\mathcal{O}_{Y}\left(E_{2}-\tau^{*} H\right)$. In this case we also have a unique non-trivial extension

$$
0 \rightarrow \mathcal{O}_{X}\left(E_{1}-\pi^{*} H\right) \rightarrow \mathcal{F} \rightarrow \mathcal{O}_{X}\left(E_{2}+E_{4}-\pi^{*} H\right) \rightarrow 0,
$$

since $\operatorname{dim} \operatorname{Ext}^{1}\left(\mathcal{O}_{X}\left(E_{2}+E_{4}\right), \mathcal{O}_{X}\left(E_{1}\right)\right)=1$ by Lemma 1.4. This extension bundle $\mathcal{F}$ is indecomposable of rank 2 with $\sigma_{*} \mathcal{F} \cong L \oplus M$. Indeed, if $\mathcal{F}$ were decomposable, then the splitting type of $\mathcal{F}\left(\pi^{*} H\right)$ would be either $\mathcal{O}_{X} \oplus$ $\mathcal{O}_{X}\left(E_{1}+E_{2}+E_{4}\right)$ or $\mathcal{O}_{X}\left(E_{i}\right) \oplus \mathcal{O}_{X}\left(E_{j}+E_{k}\right)$ with $\{i, j, k\}=\{1,2,4\}$. However, it follows from the above exact sequence that $\operatorname{Hom}\left(\mathcal{O}_{X}\left(E_{1}+E_{2}+E_{4}\right)\right.$, 
$\left.\mathcal{F}\left(\pi^{*} H\right)\right)=0$, so that the former case cannot occur. In the latter case, we apply the functor $\operatorname{Ext}^{\bullet}\left(\mathcal{O}_{X}\left(E_{j}+E_{k}-\pi^{*} H\right),-\right)$ to the exact sequence above. If $i \neq 1$ then $\operatorname{Hom}\left(\mathcal{O}_{X}\left(E_{j}+E_{k}\right), \mathcal{F}\left(\pi^{*} H\right)\right)=0$ and we get a contradiction. If $i=1$ then we have the induced exact sequence

$$
\begin{aligned}
& 0 \rightarrow \operatorname{Hom}\left(\mathcal{O}_{X}\left(E_{2}+E_{4}\right), \mathcal{F}\left(\pi^{*} H\right)\right) \rightarrow \operatorname{Hom}\left(\mathcal{O}_{X}\left(E_{2}+E_{4}\right), \mathcal{O}_{X}\left(E_{2}+E_{4}\right)\right) \\
& \quad \stackrel{\delta}{\rightarrow} \operatorname{Ext}^{1}\left(\mathcal{O}_{X}\left(E_{2}+E_{4}\right), \mathcal{O}_{X}\left(E_{1}\right)\right)
\end{aligned}
$$

with the non-zero connecting homomorphism $\delta$. It follows that $\delta$ is injective and $\operatorname{Hom}\left(\mathcal{O}_{X}\left(E_{2}+E_{4}\right), \mathcal{F}\left(\pi^{*} H\right)\right)=0$. We get a contradiction as well.

Replacing $L$ and $M$, we see that the indecomposable bundle $\mathcal{F}$ is given by another unique non-trivial extension

$$
0 \rightarrow \mathcal{O}_{X}\left(E_{2}-\pi^{*} H\right) \rightarrow \mathcal{F} \rightarrow \mathcal{O}_{X}\left(E_{1}+E_{4}-\pi^{*} H\right) \rightarrow 0 .
$$

Replacing the roles of $E_{4}$ by $E_{2}$ in the argument above we also have

$$
0 \rightarrow \mathcal{O}_{X}\left(E_{4}-\pi^{*} H\right) \rightarrow \mathcal{F} \rightarrow \mathcal{O}_{X}\left(E_{1}+E_{2}-\pi^{*} H\right) \rightarrow 0 .
$$

We denote this vector bundle $\mathcal{F}$ by $\mathcal{G}_{3}$. Similarly, we can define the indecomposable vector bundle $\mathcal{G}_{i}$ of rank 2 for $1 \leq i \leq 4$ by a unique non-trivial extension

$$
0 \rightarrow \mathcal{O}_{X}\left(E_{j}-\pi^{*} H\right) \rightarrow \mathcal{G}_{i} \rightarrow \mathcal{O}_{X}\left(E_{k}+E_{l}-\pi^{*} H\right) \rightarrow 0
$$

with any choice of $j, k, l$ such that $\{i, j, k, l\}=\{1,2,3,4\}$. Clearly the vector bundle $\mathcal{G}_{i}$ is stable under the automorphisms $\psi_{j k}, \psi_{k l}, \psi_{l j}$ introduced in Remark 3.2.

Proposition 3.4. Let $X$ be the blowup of $\mathbb{P}^{2}$ at four points in general position, $\sigma: X \rightarrow Y$ the blow-down of an exceptional curve $E_{4}$ and let $\mathcal{F}$ be an indecomposable vector bundle of rank 2 on $X$. If $\sigma_{*} \mathcal{F}$ is a Frobenius summand on $Y$, then $\mathcal{F}$ is isomorphic either to the bundle $\mathcal{G}$ given in Example 3.1, or one of $\mathcal{G}_{1}, \mathcal{G}_{2}, \mathcal{G}_{3}$ given in Example 3.3.

Proof. Since $\sigma_{*} \mathcal{F}$ is a Frobenius summand on a toric surface $Y$, it has a splitting $\sigma_{*} \mathcal{F} \cong L \oplus M$ by Lemma 1.2. We will examine the extension (3.1) with all possible types of such a splitting.

(i) Suppose first that $L \cong M$. Then

$$
\operatorname{Ext}^{1}\left(\sigma^{*}(M) \otimes \mathcal{O}_{X}\left(E_{4}\right), \sigma^{*} L\right) \cong H^{1}\left(X, \mathcal{O}_{X}\left(-E_{4}\right)\right)=0
$$

by Lemma 1.4. Thus $\mathcal{F}$ splits into a direct sum of line bundles in this case.

(ii) Suppose $\sigma_{*} \mathcal{F}$ has a free summand, say, $L \cong \mathcal{O}_{Y}$. If $\mathcal{F}$ were indecomposable, then it would be given by a non-trivial extension

$$
0 \rightarrow \mathcal{O}_{X} \rightarrow \mathcal{F} \rightarrow \sigma^{*}(M) \otimes \mathcal{O}_{X}\left(E_{4}\right) \rightarrow 0
$$


by Corollary 2.3. But this is impossible, since $\operatorname{Ext}^{1}\left(\sigma^{*}(M) \otimes \mathcal{O}_{X}\left(E_{4}\right)\right.$, $\left.\mathcal{O}_{X}\right) \cong H^{1}\left(X, \sigma^{*}\left(M^{-1}\right) \otimes \mathcal{O}_{X}\left(-E_{4}\right)\right)=0$ for all invertible Frobenius summands $M$ on $Y$ by Lemmas 1.2 and 1.4.

(iii) Suppose $\sigma_{*} \mathcal{F} \cong \mathcal{O}_{Y}\left(-\pi^{*} H\right) \oplus \mathcal{O}_{Y}\left(E_{i}-\tau^{*} H\right)$ with $i=1,2,3$. If $\mathcal{F}$ were indecomposable, then it would be given by a non-trivial extension

$$
0 \rightarrow \mathcal{O}_{X}\left(E_{i}-\pi^{*} H\right) \rightarrow \mathcal{F} \rightarrow \mathcal{O}_{X}\left(E_{4}-\pi^{*} H\right) \rightarrow 0
$$

by Corollary 2.3. But this is impossible, since $\operatorname{Ext}^{1}\left(\mathcal{O}_{X}\left(E_{4}-\pi^{*} H\right)\right.$, $\left.\mathcal{O}_{X}\left(E_{i}-\pi^{*} H\right)\right) \cong H^{1}\left(X, \mathcal{O}_{X}\left(E_{i}-E_{4}\right)\right)=0$ by Lemma 1.4.

(iv) Suppose $\sigma_{*} \mathcal{F} \cong \mathcal{O}_{Y}\left(E_{i}-\tau^{*} H\right) \oplus \mathcal{O}_{Y}\left(E^{\prime}-2 \tau^{*} H\right)$ with $i=1,2,3$. If $\mathcal{F}$ were indecomposable, then there would exist a non-trivial extension

$$
0 \rightarrow \mathcal{O}_{X}\left(E_{i}-\pi^{*} H\right) \rightarrow \mathcal{F} \rightarrow \mathcal{O}_{X}\left(E-2 \pi^{*} H\right) \rightarrow 0
$$

by Corollary 2.3. But this is impossible, since $\operatorname{Ext}^{1}\left(\mathcal{O}_{X}\left(E-2 \pi^{*} H\right)\right.$, $\left.\mathcal{O}_{X}\left(E_{i}-\pi^{*} H\right)\right) \cong H^{1}\left(X, \mathcal{O}_{X}\left(\pi^{*} H+E_{i}-E\right)\right)=0$ by Lemma 1.4.

In cases (i)-(iv) we have seen so far, any Frobenius summand $\mathcal{F}$ sitting in (3.1) splits into a direct sum of line bundles. In the remaining cases $\sigma_{*} \mathcal{F}$ is isomorphic either to $\mathcal{O}_{Y}\left(-\tau^{*} H\right) \oplus \mathcal{O}_{Y}\left(E^{\prime}-2 \tau^{*} H\right)$ or $\mathcal{O}_{Y}\left(E_{i}-\tau^{*} H\right) \oplus$ $\mathcal{O}_{Y}\left(E_{j}-\tau^{*} H\right)$ with $1 \leq i<j \leq 3$. These are exactly the cases treated in Examples 3.1 and 3.3, from which the assertion follows.

REMARK 3.5. It is not a sufficient condition for $\mathcal{F}$ to be a Frobenius summand on $X$ that so is $\sigma_{*} \mathcal{F}$ on $Y$. Actually we will see that $\mathcal{G}$ is the only indecomposable Frobenius summand of rank 2 on $X$. The reason why $\mathcal{G}_{i}$ is not a Frobenius summand is as follows: Let $\rho=\sigma_{i}: X \rightarrow Y_{i}$ be the blowdown of $E_{i}$. Then $\mathcal{G}_{i}$ descends to a vector bundle $\mathcal{G}_{i}^{\prime}=\rho_{*} \mathcal{G}_{i}$ on $Y_{i}$ such that $\mathcal{G}_{i}=\rho^{*} \mathcal{G}_{i}^{\prime}$. Since $\mathcal{G}_{i}$ is indecomposable, so is $\mathcal{G}_{i}^{\prime}$, too. Thus, $\mathcal{G}_{i}^{\prime}=\rho_{*} \mathcal{G}_{i}$ cannot be a Frobenius summand on the toric surface $Y_{i}$.

3.2. Rank 3 Frobenius summands on a 4-point blowup. We now apply Corollary 2.4 to give a candidate for an indecomposable Frobenius summands of rank 3 .

EXAMPLE 3.6. We consider case (1) of Corollary 2.4 for $L_{i}=\mathcal{O}_{Y}\left(E_{i}-\tau^{*} H\right)$ with $i=1,2,3$. Since $\operatorname{Ext}^{1}\left(\mathcal{O}_{X}\left(E_{3}+E_{4}\right), \mathcal{O}_{X}\left(E_{i}\right)\right)$ is a one-dimensional vector space for $i=1,2$ by Lemma 1.4, we have an extension

$$
\begin{aligned}
0 & \rightarrow \mathcal{O}_{X}\left(E_{1}-\pi^{*} H\right) \oplus \mathcal{O}_{X}\left(E_{2}-\pi^{*} H\right) \rightarrow \mathcal{B} \\
& \rightarrow \mathcal{O}_{X}\left(E_{3}+E_{4}-\pi^{*} H\right) \rightarrow 0
\end{aligned}
$$

given by an extension class $\varepsilon=\left(\varepsilon_{1}, \varepsilon_{2}\right)$ with $0 \neq \varepsilon_{i} \in \operatorname{Ext}^{1}\left(\mathcal{O}_{X}\left(E_{3}+E_{4}\right)\right.$, $\left.\mathcal{O}_{X}\left(E_{i}\right)\right) \cong k$. It follows as in Example 3.1 that $\sigma_{*} \mathcal{B} \cong L_{1} \oplus L_{2} \oplus L_{3}$.

We claim that this vector bundle $\mathcal{B}$ of rank 3 is indecomposable. Suppose on the contrary that $\mathcal{B}$ is decomposable. Then it has a splitting $\mathcal{B} \cong \widetilde{L_{i}} \oplus \mathcal{B}^{\prime}$ with $\widetilde{L_{i}} \cong \mathcal{O}_{X}\left(E_{i}-\pi^{*} H\right)$ or $\mathcal{O}_{X}\left(E_{i}+E_{4}-\pi^{*} H\right)$ for some $i=1,2,3$. The other 
summand $\mathcal{B}^{\prime}$ must be isomorphic to $\mathcal{G}_{i}$ or $\mathcal{O}_{X}\left(D_{1}-\pi^{*} H\right) \oplus \mathcal{O}_{X}\left(D_{2}-\pi^{*} H\right)$, where $D_{1}$ and $D_{2}$ are effective $\pi$-exceptional divisors with $D_{1}+D_{2} \leq E-E_{i}$.

In case $i=1$ or 2 , we consider the composition map

$$
\alpha: \mathcal{O}_{X}\left(E_{i}-\pi^{*} H\right) \stackrel{\imath}{\rightarrow} \mathcal{B} \rightarrow \widetilde{L_{i}},
$$

where $\imath$ is the injective map induced by (3.4) and the second surjection is a splitting map. If $\alpha \in \operatorname{Hom}\left(\mathcal{O}_{X}\left(E_{i}-\pi^{*} H\right), \widetilde{L_{i}}\right) \cong k$ is a non-zero map, then $\mathcal{O}_{X}\left(E_{i}-\pi^{*} H\right)$ is a direct summand of $\mathcal{B}$ via the map $\imath$, contradicting to our assumption that $\varepsilon_{i} \neq 0$. If $\alpha=0$, then there exists a non-zero map $\mathcal{O}_{X}\left(E_{i}-\pi^{*} H\right) \rightarrow \mathcal{B}^{\prime}$ to the other summand $\mathcal{B}^{\prime}$ of $\mathcal{B}$, but this is impossible, since $\operatorname{Hom}\left(\mathcal{O}_{X}\left(E_{i}-\pi^{*} H\right), \mathcal{B}^{\prime}\right)=0$ in any case.

In the case where $\mathcal{B} \cong \widetilde{L_{3}} \oplus \mathcal{B}^{\prime}$, we note that $\operatorname{Hom}\left(\mathcal{B}^{\prime}, \mathcal{O}_{X}\left(E_{3}+E_{4}-\right.\right.$ $\left.\left.\pi^{*} H\right)\right)=0$, so that a surjective map $\widetilde{L_{3}} \rightarrow \mathcal{O}_{X}\left(E_{3}+E_{4}-\pi^{*} H\right)$ is induced from exact sequence (3.4). Then $\widetilde{L_{3}}=\mathcal{O}_{X}\left(E_{3}+E_{4}-\pi^{*} H\right)$ and we consider the composition map

$$
\beta: \widetilde{L_{3}} \hookrightarrow \mathcal{B} \rightarrow \mathcal{O}_{X}\left(E_{3}+E_{4}-\pi^{*} H\right)
$$

instead of $\alpha$, where the first injection is a splitting map and the second surjection is that in (3.4). Arguing as before, we obtain a contradiction as well. Thus, $\mathcal{B}$ is indecomposable.

REMARK 3.7. A rank 3 indecomposable bundle $\mathcal{B}$ given in Example 3.6 is uniquely determined up to isomorphism. Indeed, let $\varepsilon=\left(\varepsilon_{1}, \varepsilon_{2}\right)$ and $\varepsilon^{\prime}=\left(\varepsilon_{1}^{\prime}, \varepsilon_{2}^{\prime}\right)$ be two extension classes with non-zero $\varepsilon_{i}, \varepsilon_{i}^{\prime} \in \operatorname{Ext}^{1}\left(\mathcal{O}_{X}\left(E_{3}+E_{4}-\right.\right.$ $\left.\left.\pi^{*} H\right), L_{i}\right) \cong k$ for $i=1,2$. Since $\varepsilon_{i}$ and $\varepsilon_{i}^{\prime}$ live in the same one-dimensional $k$-vector space, one can consider their ratio $\varepsilon_{i}^{\prime} / \varepsilon_{i} \in k^{*} \cong \operatorname{Aut}\left(L_{i}\right)$. Then the extension bundles corresponding to $\varepsilon$ and $\varepsilon^{\prime}$ are identified via the automorphism $\varepsilon_{1}^{\prime} / \varepsilon_{1} \oplus \varepsilon_{2}^{\prime} / \varepsilon_{2} \in \operatorname{Aut}\left(L_{1} \oplus L_{2}\right)$. On the other hand, if $\varepsilon_{i}=0$ for $i=1$ or 2 , then the extension bundle has a direct summand $\sigma^{*} L_{i}$ and so decomposable. It follows from the symmetry with Corollary 2.4 that $\mathcal{B}$ is a unique indecomposable bundle sitting in an exact sequence

$$
0 \rightarrow \mathcal{O}_{X}\left(E_{i}-\pi^{*} H\right) \oplus \mathcal{O}_{X}\left(E_{j}-\pi^{*} H\right) \rightarrow \mathcal{B} \rightarrow \mathcal{O}_{X}\left(E_{k}+E_{l}-\pi^{*} H\right) \rightarrow 0
$$

with any choice of indexes $i, j, k, l$ with $\{i, j, k, l\}=\{1,2,3,4\}$.

\section{The global FFRT property of a four-point blowup of $\mathbb{P}^{2}$}

We continue to work on the blowup $\pi: X \rightarrow \mathbb{P}^{2}$ at four points $P_{1}, P_{2}, P_{3}, P_{4}$ on $\mathbb{P}^{2}$ in general position. Let $E_{i}=\pi^{-1}\left(P_{i}\right)$ be the exceptional curve over $P_{i}$, let $E=E_{1}+E_{2}+E_{3}+E_{4}$ and let $H$ be a line on $\mathbb{P}^{2}$.

This section is devoted to proving the following theorem.

TheOREM 4.1. Any indecomposable Frobenius summand on the surface $X$ coincides with one of the following vector bundles of rank $\leq 3$.

(1) line bundles $\mathcal{O}_{X}, \mathcal{O}_{X}\left(E-2 \pi^{*} H\right)$ and $\mathcal{O}_{X}\left(E_{i}-\pi^{*} H\right)$ for $i=1,2,3,4$; 
(2) an indecomposable rank 2 bundle $\mathcal{G}$ given by a unique non-trivial extension

$$
0 \rightarrow \mathcal{O}_{X}\left(-\pi^{*} H\right) \rightarrow \mathcal{G} \rightarrow \mathcal{O}_{X}\left(E-2 \pi^{*} H\right) \rightarrow 0
$$

(3) an indecomposable rank 3 bundle $\mathcal{B}$ given by a non-trivial extension

$$
0 \rightarrow \mathcal{O}_{X}\left(E_{1}-\pi^{*} H\right) \oplus \mathcal{O}_{X}\left(E_{2}-\pi^{*} H\right) \rightarrow \mathcal{B} \rightarrow \mathcal{O}_{X}\left(E_{3}+E_{4}-\pi^{*} H\right) \rightarrow 0 .
$$

In particular, $X$ is GFFRT.

REMARK 4.2. The rank 2 bundle $\mathcal{G}$ in (2) is the one given in Example 3.1, whereas the rank 3 bundle $\mathcal{B}$ is the one given in Example 3.6. Theorem 4.1 is suggested by our empirical computation made in characteristic $p=2,3$.

Proof of Theorem 4.1. We begin with the following setup. Let $\sigma_{i}: X \rightarrow Y_{i}$ be the blow-down of $E_{i}$ for $i=1,2,3,4$, among which we specify

$$
\sigma=\sigma_{4}: X \rightarrow Y=Y_{4}: \text { the blow-down of } E_{4} .
$$

Let $\tau: Y \rightarrow \mathbb{P}^{2}$ be the blowup at $P_{1}, P_{2}, P_{3}$ and define the line bundles $L_{1}, L_{2}, L_{3}$ and $M_{1}, M_{2}$ on $Y$ by

$$
\begin{aligned}
L_{i} & =\mathcal{O}_{Y}\left(E_{i}-\tau^{*} H\right) & & \text { for } i=1,2,3 ; \\
M_{1} & =\mathcal{O}_{Y}\left(-\tau^{*} H\right), & & M_{2}=\mathcal{O}_{Y}\left(E^{\prime}-2 \tau^{*} H\right),
\end{aligned}
$$

where $E^{\prime}=E_{1}+E_{2}+E_{3}$. These are the non-trivial Frobenius summands on $Y$.

We first consider Frobenius summands of rank 1. We have the following line bundles that would be possibly non-trivial Frobenius summands on $X$; see Section 1.2 and Lemma 1.2:

$$
\begin{aligned}
& \mathcal{O}_{X}\left(E^{\prime}-2 \pi^{*} H\right), \mathcal{O}_{X}\left(E-2 \pi^{*} H\right), \mathcal{O}_{X}\left(-\pi^{*} H\right), \mathcal{O}_{X}\left(E_{i}-\pi^{*} H\right) \\
& \quad \text { and } \mathcal{O}_{X}\left(E_{j}+E_{4}-\pi^{*} H\right),
\end{aligned}
$$

where $i=1,2,3,4$ and $j=1,2,3$. We can rule out $\mathcal{O}_{X}\left(E^{\prime}-2 \pi^{*} H\right)$ from these candidates, since its push-forward by $\sigma_{j}(j=1,2,3)$ is not a Frobenius summand on $Y_{j}$. We can exclude $\mathcal{O}_{X}\left(E_{j}+E_{4}-\pi^{*} H\right)$ in a similar way. The line bundle $\mathcal{O}_{X}\left(-\pi^{*} H\right)$ is also ruled out from the candidates, because there is an automorphism of $X$ via which $\mathcal{O}_{X}\left(-\pi^{*} H\right)$ and $\mathcal{O}_{X}\left(E^{\prime}-2 \pi^{*} H\right)$ correspond with each other.

In order to determine Frobenius summands of rank $\geq 2$, we need the following results.

LEMmA 4.3. Let $\widetilde{L}$ and $\widetilde{M}$ be line bundles on $X$ such that the pair $(\widetilde{L}, \widetilde{M})$ is one of $\left(\sigma^{*} L_{i}, \sigma^{*} M_{j}\right),\left(\sigma^{*} L_{i}, \sigma^{*}\left(M_{j}\right) \otimes \mathcal{O}_{X}\left(E_{4}\right)\right)$ or $\left(\sigma^{*}\left(L_{i}\right) \otimes \mathcal{O}_{X}\left(E_{4}\right), \sigma^{*} M_{j}\right)$ for $1 \leq i \leq 3$ and $1 \leq j \leq 2$. Then $\operatorname{Ext}^{1}(\widetilde{L}, \widetilde{M})=0$.

Proof. This is verified with one by one computation of $\operatorname{dim} \operatorname{Ext}^{1}(\widetilde{L}, \widetilde{M})=$ $h^{1}\left(\widetilde{L}^{-1} \otimes \widetilde{M}\right)$ using Riemann-Roch; cf. Lemma 1.4 . 
Proposition 4.4. Let $\mathcal{F}$ be a vector bundle on $X$ satisfying conditions (a), (b) and (c) as in Section 2.1 with $\sigma_{*} \mathcal{F} \cong \bigoplus_{i=1}^{3} L_{i}^{\oplus a_{i}} \oplus \bigoplus_{j=1}^{2} M_{j}^{\oplus b_{j}}$. Then one has a decomposition

$$
\mathcal{F} \cong \mathcal{F}_{L} \oplus \mathcal{F}_{M}
$$

with vector bundles $\mathcal{F}_{L}$ and $\mathcal{F}_{M}$ satisfying conditions (a), (b) and (c) such that $\sigma_{*} \mathcal{F}_{L} \cong \bigoplus_{i=1}^{3} L_{i}^{\oplus a_{i}}$ and $\sigma_{*} \mathcal{F}_{M} \cong \bigoplus_{j=1}^{2} M_{j}^{\oplus b_{j}}$, respectively.

Proof. By Corollary 2.2, one has an exact sequence

$$
0 \rightarrow \mathcal{F}_{L} \rightarrow \mathcal{F} \rightarrow \mathcal{F}_{M} \rightarrow 0
$$

with vector bundles $\mathcal{F}_{L}$ and $\mathcal{F}_{M}$ satisfying conditions (a), (b) and (c) such that $\sigma_{*} \mathcal{F}_{L} \cong \bigoplus_{i=1}^{3} L_{i}^{\oplus a_{i}}$ and $\sigma_{*} \mathcal{F}_{M} \cong \bigoplus_{j=1}^{2} M_{j}^{\oplus b_{j}}$, respectively. Since $\mathcal{F}_{L}$ (resp. $\mathcal{F}_{M}$ ) is obtained by successive extensions with line bundles $\sigma^{*} L_{i}$ and $\sigma^{*}\left(L_{i}\right) \otimes \mathcal{O}_{X}\left(E_{4}\right)\left(\right.$ resp. $\sigma^{*} M_{j}$ and $\sigma^{*}\left(M_{j}\right) \otimes \mathcal{O}_{X}\left(E_{4}\right)$ ) by Lemma 2.1, it follows inductively from Lemma 4.3 that $\operatorname{Ext}^{1}\left(\mathcal{F}_{M}, \mathcal{F}_{L}\right)=0$, so that $\mathcal{F} \cong \mathcal{F}_{L} \oplus \mathcal{F}_{M}$.

It follows that any indecomposable Frobenius summand of rank $\geq 2$ on $X$ is either of type $\mathcal{F}_{L}$ or $\mathcal{F}_{M}$ in Proposition 4.4. We first determine the structure of the Frobenius summand $\mathcal{F}_{M}$.

Proposition 4.5. Let $\mathcal{F}$ be an indecomposable Frobenius summand of rank $r=r_{1}+r_{2} \geq 2$ such that $\sigma_{*} \mathcal{F} \cong M_{1}^{\oplus r_{1}} \oplus M_{2}^{\oplus r_{2}}$. Then $r_{1}=r_{2}=1$ and $\mathcal{F} \cong \mathcal{G}$.

Proof. It is easy to see that $r_{i}>0(i=1,2)$ if $\mathcal{F}$ is indecomposable. Indeed, if $\sigma_{*} \mathcal{F} \cong M^{\oplus r}$ for a line bundle $M$, then $\mathcal{F}$ would decompose into line bundles $\sigma^{*} M$ and $\sigma^{*}(M) \otimes \mathcal{O}_{X}\left(E_{4}\right)$. This follows inductively from Lemmas 2.1 and 1.4 as in case (i) in the proof of Proposition 3.4.

Let $\mathcal{F}_{i_{1}, i_{2}}$ denote the saturation of $\sigma^{*} M_{1}^{\oplus i_{1}} \oplus \sigma^{*} M_{2}^{\oplus i_{2}}$ in $\mathcal{F}$. Then we have an exact sequence

$$
0 \rightarrow \mathcal{F}_{r_{1}, r_{2}-1} \rightarrow \mathcal{F} \rightarrow \mathcal{O}_{X}\left(E-2 \pi^{*} H\right) \rightarrow 0
$$

by Lemma 2.1. If $r_{2} \geq 2$, then we also have

$$
0 \rightarrow \mathcal{F}_{r_{1}, r_{2}-2} \rightarrow \mathcal{F}_{r_{1}, r_{2}-1} \rightarrow \overline{M_{2}} \rightarrow 0,
$$

where $\overline{M_{2}} \cong \sigma^{*} M_{2}$ or $\sigma^{*}\left(M_{2}\right) \otimes \mathcal{O}_{X}\left(E_{4}\right)=\mathcal{O}_{X}\left(E-2 \pi^{*} H\right)$ with $E=E_{1}+$ $E_{2}+E_{3}+E_{4}$. If $\overline{M_{2}} \cong \sigma^{*} M_{2}$, then the above exact sequence splits, that is, $\mathcal{F}_{r_{1}, r_{2}-1} \cong \mathcal{F}_{r_{1}, r_{2}-2} \oplus \sigma^{*} M_{2}$. Thus exact sequence (4.1) turns out to be

$$
0 \rightarrow \mathcal{F}_{r_{1}, r_{2}-2} \oplus \sigma^{*} M_{2} \rightarrow \mathcal{F} \rightarrow \mathcal{O}_{X}\left(E-2 \pi^{*} H\right) \rightarrow 0 .
$$

However, since $\operatorname{Ext}^{1}\left(\mathcal{O}_{X}\left(E-2 \pi^{*} H\right), \sigma^{*} M_{2}\right)=H^{1}\left(X, \mathcal{O}_{X}\left(-E_{4}\right)\right)=0$ by Lemma 1.4, $\mathcal{F}$ has a direct summand $\sigma^{*} M_{2}$ in this case, contradicting to 
the indecomposability of $\mathcal{F}$. Thus, $\overline{M_{2}} \cong \mathcal{O}_{X}\left(E-2 \pi^{*} H\right)$. Using a commutative diagram as in the proof of Corollary 2.2, we see that $\mathcal{F}$ sits in another exact sequence

$$
0 \rightarrow \mathcal{F}_{r_{1}, r_{2}-2} \rightarrow \mathcal{F} \rightarrow \mathcal{O}_{X}\left(E-2 \pi^{*} H\right)^{\oplus 2} \rightarrow 0 .
$$

Repeating this procedure inductively, we obtain an exact sequence

$$
0 \rightarrow \mathcal{F}_{r_{1}, 0} \rightarrow \mathcal{F} \rightarrow \mathcal{O}_{X}\left(E-2 \pi^{*} H\right)^{\oplus r_{2}} \rightarrow 0 .
$$

As we have seen in the beginning of the proof, $\mathcal{F}_{r_{1}, 0}$ is a direct sum of copies of line bundles $\sigma^{*} M_{1}=\mathcal{O}_{X}\left(-\pi^{*} H\right)$ and $\mathcal{O}_{X}\left(E_{4}-\pi^{*} H\right)$, and if $\mathcal{F}_{r_{1}, 0}$ has a direct summand $\mathcal{O}_{X}\left(E_{4}-\pi^{*} H\right)$, then so does $\mathcal{F}$, since $\operatorname{Ext}^{1}\left(\mathcal{O}_{X}\left(E-2 \pi^{*} H\right)\right.$, $\left.\mathcal{O}_{X}\left(E_{4}-\pi^{*} H\right)\right)=0$ again by Lemma 1.4. Thus, we have an exact sequence

$$
0 \rightarrow \mathcal{O}_{X}\left(-\pi^{*} H\right)^{\oplus r_{1}} \rightarrow \mathcal{F} \rightarrow \mathcal{O}_{X}\left(E-2 \pi^{*} H\right)^{\oplus r_{2}} \rightarrow 0 .
$$

Since $\operatorname{Ext}^{1}\left(\mathcal{O}_{X}\left(E-2 \pi^{*} H\right), \mathcal{O}_{X}\left(-\pi^{*} H\right)\right)$ is a 1-dimensional vector space, the above exact sequence is given by an "extension matrix" $\varepsilon_{M}$ of size $r_{2} \times r_{1}$, to which we can make row and column elementary transformations without changing the isomorphism class of the extension bundle $\mathcal{F}$. If $r_{1} \neq r_{2}$ or rank $\varepsilon_{M}<r_{1}=r_{2}$, then $\mathcal{F}$ has a line bundle summand. If $\operatorname{rank} \varepsilon_{M}=r_{1}=r_{2}$, then $\varepsilon_{M}$ is transformed to the identity matrix of size $r_{1}=r_{2}=r / 2$ and $\mathcal{F} \cong \mathcal{G}^{\oplus r / 2}$. It follows from the indecomposability of $\mathcal{F}$ that $r=2$ and $\mathcal{F} \cong \mathcal{G}$.

We now consider the structure of the Frobenius summand $\mathcal{F}_{L}$ in Proposition 4.4. We put $\mathcal{L}=\mathcal{O}_{X}\left(-\pi^{*} H\right)=\pi^{*} \mathcal{O}_{\mathbb{P}^{2}}(-1)$ and consider a vector bundle $\mathcal{F}$ of rank $r=a_{1}+a_{2}+a_{3}$ on $X$ satisfying the following condition:

$$
\begin{aligned}
\sigma_{*} \mathcal{F} & \cong L_{1}^{\oplus a_{1}} \oplus L_{2}^{\oplus a_{2}} \oplus L_{3}^{\oplus a_{3}} \quad \text { and } \\
\operatorname{det} \mathcal{F} & =\mathcal{L}^{r}\left(a_{1} E_{1}+a_{2} E_{2}+a_{3} E_{3}+a_{4} E_{4}\right) .
\end{aligned}
$$

Lemma 4.6. Suppose that $\mathcal{F}$ is a Frobenius summand satisfying condition $(*)$ as above.

(1) $\left(\sum_{i=1}^{4} a_{i}\right)-a_{j} \leq r$ for all $j=1,2,3,4$.

(2) If $\mathcal{F}$ is indecomposable with $r \geq 2$, then $0<a_{4} \leq a_{j}$ for all $j=1,2,3$.

Proof. (1) First note that condition $(*)$ implies $\pi_{*} \mathcal{F} \cong \mathcal{O}_{\mathbb{P}^{2}}(-1)^{\oplus r}$, so that its push-forward $\left(\sigma_{j}\right)_{*} \mathcal{F}$ by $\sigma_{j}$, which is a Frobenius summand on a three-point blowup $Y_{j}$, is isomorphic to a direct sum of $r$ copies of line bundles $\mathcal{O}_{Y_{j}}\left(-\tau_{j}^{*} H\right)$ and $\mathcal{O}_{Y_{j}}\left(E_{i}-\tau_{j}^{*} H\right)$ with $i \neq j$. Since $\operatorname{det}\left(\sigma_{j}\right)_{*} \mathcal{F}=\mathcal{O}_{Y_{j}}\left(\sum_{i \neq j} a_{i} E_{i}-r \tau_{j}^{*} H\right)$, we see that $\sum_{i \neq j} a_{i} \leq r$. Since this is an equality for $j=4$, we have $a_{j} \geq a_{4}$ for $j=1,2,3$.

(2) If $a_{4}=0$, then $\mathcal{F}=\sigma^{*} \sigma_{*} \mathcal{F} \cong \bigoplus_{i=1}^{3} \mathcal{L}\left(E_{i}\right)^{\oplus a_{i}}$ splits into a direct sum of line bundles. 
REMARK 4.7. In order to prove and apply Lemma 4.6, we need the condition that the points $P_{1}, P_{2}, P_{3}, P_{4}$ are in general position. Indeed, it guarantees that $Y_{j}$ and especially $Y$ are all isomorphic to the projective plane blown up at three points that are not collinear, to which Lemma 1.2 is applicable.

Proposition 4.8. Suppose that $\mathcal{F}$ is an indecomposable Frobenius summand of rank $r \geq 2$ satisfying condition $(*)$. Then $\mathcal{F} \cong \mathcal{B}$.

Proof. First, note that $0<a_{4} \leq a_{3}$ by Lemma 4.6. We denote by $\mathcal{F}_{i_{1}, i_{2}, i_{3}}$ the saturation of $\sigma^{*}\left(L_{1}^{\oplus i_{1}} \oplus L_{2}^{\oplus i_{2}} \oplus L_{3}^{\oplus i i_{3}}\right)$ in $\mathcal{F}$, and argue as in the proof of Proposition 4.5. First, we have an exact sequence

$$
0 \rightarrow \mathcal{F}_{a_{1}, a_{2}, a_{3}-1} \rightarrow \mathcal{F} \rightarrow \mathcal{L}\left(E_{3}+E_{4}\right) \rightarrow 0
$$

by Lemma 2.1. If $a_{3} \geq 2$, then we also have

$$
0 \rightarrow \mathcal{F}_{a_{1}, a_{2}, a_{3}-2} \rightarrow \mathcal{F}_{a_{1}, a_{2}, a_{3}-1} \rightarrow \overline{L_{3}} \rightarrow 0,
$$

where $\overline{L_{3}} \cong \mathcal{L}\left(E_{3}\right)$ or $\mathcal{L}\left(E_{3}+E_{4}\right)$. If $\overline{L_{3}} \cong \mathcal{L}\left(E_{3}\right)$, then the above exact sequence splits, i.e., $\mathcal{F}_{a_{1}, a_{2}, a_{3}-1} \cong \mathcal{F}_{a_{1}, a_{2}, a_{3}-2} \oplus \mathcal{L}\left(E_{3}\right)$. Thus exact sequence (4.2) turns out to be

$$
0 \rightarrow \mathcal{F}_{a_{1}, a_{2}, a_{3}-2} \oplus \mathcal{L}\left(E_{3}\right) \rightarrow \mathcal{F} \rightarrow \mathcal{L}\left(E_{3}+E_{4}\right) \rightarrow 0 .
$$

However, since $\operatorname{Ext}^{1}\left(\mathcal{L}\left(E_{3}+E_{4}\right), \mathcal{L}\left(E_{3}\right)\right)=0$ by Lemma 1.4, the extension bundle $\mathcal{F}$ obtained as above has a direct summand $\mathcal{L}\left(E_{3}\right)$, contradicting to the indecomposability of $\mathcal{F}$. Thus $\overline{L_{3}} \cong \mathcal{L}\left(E_{3}+E_{4}\right)$. Using a commutative diagram as in the proof of Corollary 2.2, we see that $\mathcal{F}$ sits in another exact sequence

$$
0 \rightarrow \mathcal{F}_{a_{1}, a_{2}, a_{3}-2} \rightarrow \mathcal{F} \rightarrow \mathcal{L}\left(E_{3}+E_{4}\right)^{\oplus 2} \rightarrow 0 .
$$

Repeating this procedure inductively, we obtain an exact sequence

$$
0 \rightarrow \mathcal{F}_{a_{1}, a_{2}, a_{3}-a_{4}} \rightarrow \mathcal{F} \rightarrow \mathcal{L}\left(E_{3}+E_{4}\right)^{\oplus a_{4}} \rightarrow 0 .
$$

Since $\mathcal{F}^{\prime}=\mathcal{F}_{a_{1}, a_{2}, a_{3}-a_{4}}$ is the saturation of $L_{1}^{\oplus a_{1}} \oplus L_{2}^{\oplus a_{2}} \oplus L_{3}^{\oplus a_{3}-a_{4}}$ in $\mathcal{F}, \quad \sigma_{*} \mathcal{F}^{\prime}=L_{1}^{\oplus a_{1}} \oplus L_{2}^{\oplus a_{2}} \oplus L_{3}^{\oplus a_{3}-a_{4}}$ and $\operatorname{det} \mathcal{F}^{\prime}=\mathcal{L}^{r-a_{4}}\left(a_{1} E_{1}+a_{2} E_{2}+\right.$ $\left.\left(a_{3}-a_{4}\right) E_{3}\right)$. It then follows that $\mathcal{F}^{\prime}=\sigma^{*} \sigma_{*} \mathcal{F}^{\prime} \cong \mathcal{L}\left(E_{1}\right)^{\oplus a_{1}} \oplus \mathcal{L}\left(E_{2}\right)^{\oplus a_{2}} \oplus$ $\mathcal{L}\left(E_{3}\right)^{\oplus a_{3}-a_{4}}$. Namely, exact sequence (4.3) turns out to be

$$
0 \rightarrow \mathcal{L}\left(E_{1}\right)^{\oplus a_{1}} \oplus \mathcal{L}\left(E_{2}\right)^{\oplus a_{2}} \oplus \mathcal{L}\left(E_{3}\right)^{\oplus a_{3}-a_{4}} \rightarrow \mathcal{F} \rightarrow \mathcal{L}\left(E_{3}+E_{4}\right)^{\oplus a_{4}} \rightarrow 0 .
$$

If $a_{3}>a_{4}$, then $\mathcal{F}$ must have a line bundle summand $\mathcal{L}\left(E_{3}\right)$ again by the vanishing $\operatorname{Ext}^{1}\left(\mathcal{L}\left(E_{3}+E_{4}\right), \mathcal{L}\left(E_{3}\right)\right)=0$. Hence $a_{3}=a_{4}$, and by the same reason $a_{1}=a_{2}=a_{4}$. Thus, letting $a=a_{1}=a_{2}=a_{3}=a_{4}$ we have come to an exact sequence

$$
0 \rightarrow \mathcal{L}\left(E_{1}\right)^{\oplus a} \oplus \mathcal{L}\left(E_{2}\right)^{\oplus a} \rightarrow \mathcal{F} \rightarrow \mathcal{L}\left(E_{3}+E_{4}\right)^{\oplus a} \rightarrow 0 .
$$


Since $\operatorname{Ext}^{1}\left(\mathcal{L}\left(E_{3}+E_{4}\right), \mathcal{L}\left(E_{i}\right)\right)$ is a 1 -dimensional vector space for $i=1,2$, the above exact sequence is given by an "extension matrix"

$$
\varepsilon_{L}=\left[\frac{\varepsilon_{1}}{\varepsilon_{2}}\right]
$$

where $\varepsilon_{i}$ is an $a \times a$-matrix with entries in $\operatorname{Ext}^{1}\left(\mathcal{L}\left(E_{3}+E_{4}\right), \mathcal{L}\left(E_{i}\right)\right) \cong k$. If either one of $\varepsilon_{i}$ is of rank $<a$, then $\mathcal{F}$ has a line bundle summand isomorphic to $\mathcal{L}\left(E_{i}\right)$, contradicting to the indecomposability of $\mathcal{F}$. Thus $\operatorname{rank} \varepsilon_{1}=\operatorname{rank} \varepsilon_{2}=$ $a$ and both $\varepsilon_{1}$ and $\varepsilon_{2}$ are transformed to the identity matrix. It then follows that $\mathcal{F} \cong \mathcal{B}^{\oplus a}$, and the indecomposability of $\mathcal{F}$ implies that $a=1$.

In conclusion, any indecomposable Frobenius summand on $X$ is either one of those listed in Theorem 4.1 .

\section{Determination of the Frobenius summands on the four-point blowup of $\mathbb{P}^{2}$}

5.1. Observation. We again work on the blowup $\pi: X \rightarrow \mathbb{P}^{2}$ at four points in general position as in Section 4 , but we will use the notation $L_{i}$ in a different meaning in this section. Namely, let $L_{0}=\mathcal{O}_{X}\left(E_{1}+E_{2}+E_{3}+E_{4}-2 \pi^{*} H\right)$ and $L_{i}=\mathcal{O}_{X}\left(E_{i}-\pi^{*} H\right)$ for $i=1,2,3,4$. We know that exactly one $\mathcal{O}_{X}$ appears as a direct summand of $F_{*}^{e} \mathcal{O}_{X}$ by Proposition 1.1, but we have not yet proven in Section 4 that $L_{i}, \mathcal{G}$ and $\mathcal{B}$ are really Frobenius summands on $X$. In this section, we will verify this to be true.

Thanks to Theorem 4.1, we can write

$$
F_{*}^{e} \mathcal{O}_{X} \cong \mathcal{O}_{X} \oplus \bigoplus_{i=0}^{4} L_{i}^{\oplus a_{e}} \oplus \mathcal{B}^{\oplus b_{e}} \oplus \mathcal{G}^{\oplus c_{e}},
$$

where $0 \leq a_{e}, b_{e}, c_{e} \in \mathbb{Z}$. (Note that the numbers of copies of $L_{i}$ 's are equal since they are transformed to each other via automorphisms of $X$.) Comparing the push-forwards by $\sigma=\sigma_{4}$ (or, ranks and the first Chern classes; note that the first Chern class of $F_{*}^{e} \mathcal{O}_{X}$ is $\frac{p^{2 e}-p^{e}}{2} K_{X}$; see $\left.[\mathrm{KS}]\right)$ of the both sides we deduce the following equalities.

$$
a_{e}+b_{e}=p^{e}-1 \quad \text { and } \quad a_{e}+c_{e}=\frac{\left(p^{e}-1\right)\left(p^{e}-2\right)}{2} .
$$

THEOREM 5.1. In the notation as above, one has

$$
a_{e}=p^{e}-2, \quad b_{e}=1 \quad \text { and } \quad c_{e}=\frac{\left(p^{e}-2\right)\left(p^{e}-3\right)}{2}
$$

for all $e \geq 1$. 
Proof. Let $q=p^{e}$ and let $L:=L_{1}=\mathcal{O}_{X}\left(E_{1}-\pi^{*} H\right)$. Our goal is to show that $a_{e}=q-2$, or equivalently that $F_{*}^{e} \mathcal{O}_{X}$ has exactly $q-2$ copies of $L$ as its direct summands. To this end, we will look at the natural pairing

$$
\operatorname{Hom}\left(L, F_{*}^{e} \mathcal{O}_{X}\right) \times \operatorname{Hom}\left(F_{*}^{e} \mathcal{O}_{X}, L\right) \rightarrow \operatorname{Hom}(L, L)=k .
$$

Let $x=x_{1} / x_{0}, y=x_{2} / x_{0}$ be the affine coordinates at $P_{1}=(1: 0: 0) \in \mathbb{P}^{2}$. Then

$$
\begin{aligned}
\operatorname{Hom}\left(L, F_{*}^{e} \mathcal{O}_{X}\right) & \cong H^{0}\left(X, F_{*}^{e}\left(L^{-q}\right)\right) \\
& =H^{0}\left(X, L^{-q}\right)=H^{0}\left(X, \mathcal{O}_{X}\left(q \pi^{*} H-q E_{1}\right)\right)
\end{aligned}
$$

is identified with the subspace of $H^{0}\left(\mathbb{P}^{2}, \mathcal{O}_{\mathbb{P}^{2}}(q H)\right)=\left\langle x^{i} y^{j} \mid 0 \leq i, j ; i+j \leq q\right\rangle$ consisting of polynomials of order $\geq q$ at the point $P_{1}$, which is exactly equal to

$$
V=\left\langle x^{q}, x^{q-1} y, \ldots, y^{q}\right\rangle .
$$

On the other hand, it follows from the adjunction formula that

$$
\begin{aligned}
\mathcal{H o m}\left(F_{*}^{e} \mathcal{O}_{X}, L\right) & \cong \mathcal{H} \operatorname{om}\left(F_{*}^{e} \mathcal{O}_{X}, \omega_{X}\right) \otimes \omega_{X}^{-1} \otimes L \\
& \cong F_{*}^{e}\left(\omega_{X}\right) \otimes \omega_{X}^{-1} \otimes L \cong F_{*}^{e}\left(\omega_{X}^{1-q} \otimes L^{q}\right) \\
& \cong F_{*}^{e} \mathcal{O}_{X}\left((2 q-3) \pi^{*} H+E_{1}-(q-1)\left(E_{2}+E_{3}+E_{4}\right)\right) .
\end{aligned}
$$

Thus, $\operatorname{Hom}\left(F_{*}^{e} \mathcal{O}_{X}, L\right) \cong H^{0}\left(X, \mathcal{O}_{X}\left((2 q-3) \pi^{*} H-(q-1)\left(E_{2}+E_{3}+E_{4}\right)\right)\right)$ and this is identified with

$$
W=\left\langle(x-1)^{i}(y-1)^{j} \mid i, j \leq q-2, i+j \geq q-1\right\rangle
$$

by a similar argument as above with respect to affine coordinates $x-1, y-1$ at $P_{4}=(1: 1: 1) \in \mathbb{P}^{2}$. The pairing (5.1) is identified with

$$
\begin{aligned}
H^{0}\left(X, F_{*}^{e}\left(L^{-q}\right)\right) \times H^{0}\left(X, F_{*}^{e}\left(\omega_{X}^{1-q} \otimes L^{q}\right)\right) & \stackrel{\mu}{\rightarrow} H^{0}\left(X, F_{*}^{e}\left(\omega_{X}^{1-q}\right)\right) \\
& \stackrel{t_{e}}{\rightarrow} H^{0}\left(X, \mathcal{O}_{X}\right),
\end{aligned}
$$

where $\mu$ is the multiplication map and $t_{e}$ is the map induced by the trace map (or the Cartier operator) $\operatorname{Tr}_{e}: F_{*}^{e} \omega_{X} \rightarrow \omega_{X}$. Furthermore, it is identified with the pairing $\langle-,-\rangle: V \times W \rightarrow k$ given by

$$
\langle\varphi, \psi\rangle=\text { the coefficient of } \varphi \psi \text { in }(x y)^{q-1}
$$

for $\varphi \in V$ and $\psi \in W$; see, for example, [Sch, Observation 3.5]. Hence, $L$ is a direct summand of $F_{*}^{e} \mathcal{O}_{X}$ via $\varphi \in V$ if and only if there exists $\psi \in W$ such that $\langle\varphi, \psi\rangle=1$. Now let

$$
\begin{aligned}
& \operatorname{Ann}_{V}(W)=\{\varphi \in V \mid\langle\varphi, \psi\rangle=0 \text { for all } \psi \in W\}, \\
& \operatorname{Ann}_{W}(V)=\{\psi \in W \mid\langle\varphi, \psi\rangle=0 \text { for all } \varphi \in V\}
\end{aligned}
$$


and let $\bar{V}=V / \operatorname{Ann}_{V}(W), \bar{W}=W / \operatorname{Ann}_{W}(V)$. Then we have an induced perfect pairing $\bar{V} \times \bar{W} \rightarrow k$ and $\operatorname{dim} \bar{V}=\operatorname{dim} \bar{W}$ is equal to the rank of a $\frac{(q-1)(q-2)}{2} \times(q-1)$-matrix

$$
A^{(e)}=\left[\left(\begin{array}{c}
i \\
l-1
\end{array}\right)\left(\begin{array}{c}
j \\
q-1-l
\end{array}\right)\right]_{(i, j) \in I, 1 \leq l \leq q-1},
$$

whose rows are indexed by $(i, j) \in I=\left\{(i, j) \in \mathbb{Z}^{2} \mid 1 \leq i, j \leq q-2, i+j \geq q-1\right\}$ and columns by $l=1,2, \ldots, q-1$. Each entry of $A^{(e)}$ is a product of binomial coefficients that is equal to $\left\langle x^{q-l} y^{l},(x-1)^{i}(y-1)^{j}\right\rangle \in \mathbb{F}_{p}$.

Recall that $a_{e} \geq 0$ is the maximum integer such that $L^{\oplus a_{e}}$ is isomorphic to a direct summand of $F_{*}^{e} \mathcal{O}_{X}$.

Claim 5.1. $a_{e}=\operatorname{rank} A^{(e)}$.

Proof. Let $r=\operatorname{rank} A^{(e)}$ and choose $\varphi_{1}, \ldots, \varphi_{r} \in V$ that reduce to a basis $\bar{\varphi}_{1}, \ldots, \bar{\varphi}_{r}$ of $\bar{V}$. Then $\varphi_{1}, \ldots, \varphi_{r}$ are linearly independent over $k\left(x^{q}, y^{q}\right)$, since $\bar{V}$ is spanned by images of $x^{q-1} y, \ldots, x y^{q-1}$. Hence, $\varphi_{1}, \ldots, \varphi_{r}$ give an injective $\mathcal{O}_{X}$-module homomorphism $\varphi: L^{\oplus r} \hookrightarrow F_{*}^{e} \mathcal{O}_{X}$. Next, choose $\psi_{1}, \ldots, \psi_{r} \in W$ that reduce to the dual basis $\bar{\varphi}_{1}^{*}, \ldots, \bar{\varphi}_{r}^{*}$ of $\bar{W}=\bar{V}^{*}$ and let $\psi: F_{*}^{e} \mathcal{O}_{X} \rightarrow L^{\oplus r}$ be the $\mathcal{O}_{X}$-module homomorphism given by them. Then one has $\left\langle\varphi_{i}, \psi_{j}\right\rangle=\left\langle\bar{\varphi}_{i}, \bar{\varphi}_{j}^{*}\right\rangle=\delta_{i j}$ for $1 \leq i, j \leq r$, so that $\psi \circ \varphi$ is the identity on $L^{\oplus r}$. Thus, $r \leq a_{e}$.

To complete the proof of the claim, suppose to the contrary that $r<a:=a_{e}$. Choose $\varphi_{1}, \ldots, \varphi_{a} \in V$ and $\psi_{1}, \ldots, \psi_{a} \in W$ so that the composition of the corresponding maps $\varphi: L^{\oplus a} \hookrightarrow F_{*}^{e} \mathcal{O}_{X}$ and $\psi: F_{*}^{e} \mathcal{O}_{X} \rightarrow L^{\oplus a}$ is the identity on $L^{\oplus a}$. Then one has that $\left\langle\bar{\varphi}_{i}, \bar{\psi}_{j}\right\rangle=\left\langle\varphi_{i}, \psi_{j}\right\rangle=\delta_{i j}$ for $1 \leq i, j \leq a$, that is, the $a \times a$-matrix with $(i, j)$-entries $\left\langle\bar{\varphi}_{i}, \bar{\psi}_{j}\right\rangle$ is the identity matrix. But this is impossible since $\bar{\varphi}_{1}, \ldots, \bar{\varphi}_{a}$ are linearly dependent.

Thus, the theorem follows from the following lemma.

Lemma 5.2. $\operatorname{rank} A^{(e)}=p^{e}-2$.

Proof (Induction on e). Let $q=p^{e}$ as before. For $(i, j) \in I$, we denote the $(i, j)$ th row vector of $A^{(e)}$ by

$$
\begin{aligned}
v_{i, j}^{(e)}= & {\left[\left(\begin{array}{c}
i \\
0
\end{array}\right)\left(\begin{array}{c}
j \\
q-2
\end{array}\right),\left(\begin{array}{l}
i \\
1
\end{array}\right)\left(\begin{array}{c}
j \\
q-3
\end{array}\right), \ldots,\right.} \\
& \left.\left(\begin{array}{c}
i \\
l-1
\end{array}\right)\left(\begin{array}{c}
j \\
q-1-l
\end{array}\right), \ldots,\left(\begin{array}{c}
i \\
q-2
\end{array}\right)\left(\begin{array}{l}
j \\
0
\end{array}\right)\right],
\end{aligned}
$$

where we follow the convention on the binomial coefficients that $\left(\begin{array}{c}m \\ n\end{array}\right)=0$ if $m<n$. We divide $A^{(e)}$ into $q-2$ row-blocks according to the value of $i+j=q-1, q, \ldots, 2 q-4$. Then we have the following relation between rows in $(i+j)$ - and $(i+j+1)$-blocks.

$$
(i+1) v_{i, j}^{(e)}+j v_{i+1, j-1}^{(e)}=(i+j-q+3) v_{i+1, j}^{(e)}=(i+j+3) v_{i+1, j}^{(e)} .
$$


Indeed, it is deduced from the following easy to check equality in $\mathbb{Z}$.

$$
\begin{aligned}
& (i+1)\left(\begin{array}{c}
i \\
l-1
\end{array}\right)\left(\begin{array}{c}
j \\
q-1-l
\end{array}\right)+j\left(\begin{array}{l}
i+1 \\
l-1
\end{array}\right)\left(\begin{array}{c}
j-1 \\
q-1-l
\end{array}\right) \\
& =(i+j-q+3)\left(\begin{array}{l}
i+1 \\
l-1
\end{array}\right)\left(\begin{array}{c}
j \\
q-1-l
\end{array}\right) .
\end{aligned}
$$

Since the coefficient of the right-hand side satisfies $2 \leq i+j-q+3 \leq q-1$, it is not zero in $\mathbb{F}_{p}$ if $q=p$ is prime. It follows that all the row vectors of $A^{(1)}$ are linear combinations (over $\mathbb{F}_{p}$ ) of $p-2$ linearly independent vectors

$$
\begin{aligned}
& v_{1, p-2}^{(1)}=[1, p-2,0, \ldots, 0], \\
& v_{2, p-3}^{(1)}=[0,2, p-3,0, \ldots, 0], \quad \ldots, \\
& v_{p-2,1}^{(1)}=[0, \ldots 0, p-2,1] .
\end{aligned}
$$

Thus, the claim holds true if $e=1$.

Now we consider the following subspaces of the $k$-vector space of all the row vectors of length $p^{e}-1$ with entries in $k$.

$V^{(e)}$ : the subspace spanned by the row vectors of $A^{(e)}$.

$W^{(e)}$ : the subspace consisting of the row vectors whose $l p$ th entry is zero for all $l=1, \ldots, p^{e-1}-1$.

(The notation $V^{(e)}, W^{(e)}$ here is independent of the notation $V, W$ in the proof of Theorem 5.1.) Clearly, $\operatorname{dim} W^{(e)}=p^{e}-p^{e-1}$, and $\operatorname{dim} V^{(e-1)}=$ $p^{e-1}-2$ by induction. Therefore, the lemma follows immediately from the following

Claim 5.2. Let $q=p^{e}$ with $e \geq 2$. Then

(1) $W^{(e)}=\left\langle v_{1, q-2}^{(e)}, v_{2, q-3}^{(e)}, \ldots, v_{q-2,1}^{(e)}\right\rangle \subset V^{(e)}$.

(2) $V^{(e)} / W^{(e)} \cong V^{(e-1)}$.

Assertion (1) of the claim follows from

$$
\begin{aligned}
& v_{1, q-2}^{(e)}=[1, q-2,0, \ldots, 0], \\
& v_{2, q-3}^{(e)}=[0,2, q-3,0, \ldots, 0], \\
& v_{q-2,1}^{(e)}=[0, \ldots 0, q-2,1] .
\end{aligned}
$$

To prove $(2)$, we calculate the $(n, m ; l p)$-entry

$$
\left(\begin{array}{c}
n \\
l p-1
\end{array}\right)\left(\begin{array}{c}
m \\
p^{e}-1-l p
\end{array}\right) \bmod p
$$

of $A^{(e)}$ for $l=1, \ldots, p^{e-1}-1$. Looking at the rational expression of the binomial coefficient

$$
\left(\begin{array}{c}
n \\
l p-1
\end{array}\right)=\frac{n(n-1) \cdots(n-l p+2)}{(l p-1)(l p-2) \cdots 2 \cdot 1}
$$


and comparing the numerator and the denominator with respect to the multiplicities of the prime divisor $p$ and the factors prime to $p$, we see that

$$
\left(\begin{array}{c}
n \\
l p-1
\end{array}\right) \equiv \begin{cases}\left(\begin{array}{c}
i \\
l-1
\end{array}\right) & \text { if } n=i p+p-1 \equiv-1 \\
0 & \text { otherwise }\end{cases}
$$

Similarly,

$$
\left(\begin{array}{c}
m \\
p^{e}-1-l p
\end{array}\right) \equiv\left\{\begin{array}{cl}
\left(\begin{array}{c}
j \\
p^{e-1}-1-l
\end{array}\right) & \text { if } m=j p+p-1 \equiv-1 \\
0 & \text { otherwise }
\end{array} \quad(\bmod p) .\right.
$$

Hence, the $(i p+p-1, j p+p-1 ; l p)$-entry of $A^{(e)}$ is equal to the $(i, j ; l)$-entry of $A^{(e-1)}$ and all the other entries in the $l p$ th column are zero. Thus, assertion (2) is proved.

REMARK 5.3. The direct summand $L^{\oplus p^{e-1}-2}$ of $F_{*}^{e} \mathcal{O}_{X}$ corresponding to $V^{(e)} / W^{(e)} \cong V^{(e-1)}$ comes from the composition of the splitting injective maps $L^{\oplus p^{e-1}-2} \hookrightarrow F_{*}^{e-1} \mathcal{O}_{X}$ and $F_{*}^{e-1} \mathcal{O}_{X} \hookrightarrow F_{*}^{e} \mathcal{O}_{X}$.

Corollary 5.4. Let the notation be as in Theorem 4.1. Then for any power $q=p^{e}$ of the characteristic $p$ with $e \geq 1$ one has

$$
\begin{aligned}
F_{*}^{e} \mathcal{O}_{X} & \cong \mathcal{O}_{X} \oplus \bigoplus_{i=0}^{4} L_{i}^{\oplus(q-2)} \oplus \mathcal{B} \oplus \mathcal{G}^{\oplus \frac{(q-2)(q-3)}{2}}, \\
F_{*}^{e} L_{i} & \cong L_{i}^{\oplus q} \oplus \mathcal{G}^{\oplus \frac{q(q-1)}{2}} \quad \text { for } 0 \leq i \leq 4, \\
F_{*}^{e} \mathcal{B} & \cong \bigoplus_{i=0}^{4} L_{i}^{\oplus q} \oplus \mathcal{G}^{\oplus \frac{q(3 q-5)}{2}} \quad \text { and } \quad F_{*}^{e} \mathcal{G} \cong \mathcal{G}^{\oplus q^{2}} .
\end{aligned}
$$

Proof. The formula for $F_{*}^{e} \mathcal{O}_{X}$ is an immediate consequence of Theorems 4.1 and 5.1.

In order to show the formula for $F_{*}^{e} L_{i}$ we may assume that $i=0$ by symmetry; cf. Remark 3.2. Since $\mathcal{O}_{X}$ and $\mathcal{B}$ are not direct summands of $F_{*}^{e} L_{0}$ by Theorem 5.1, we can write $F_{*}^{e} L_{0} \cong L_{0}^{\oplus a} \oplus \bigoplus_{i=1}^{4} L_{i}^{\oplus b} \oplus \mathcal{G}^{\oplus c}$ again by symmetry. Pushing it out by the blow-down $\sigma: X \rightarrow Y$ of $E_{4}$, one sees that

$$
\begin{aligned}
& F_{*}^{e} \mathcal{O}_{Y}\left(E^{\prime}-2 \tau^{*} H\right) \\
& \quad \cong \mathcal{O}_{Y}\left(-\tau^{*} H\right)^{\oplus b+c} \oplus \bigoplus_{i=1}^{3} \mathcal{O}_{Y}\left(E_{i}-\tau^{*} H\right)^{\oplus b} \oplus \mathcal{O}_{Y}\left(E^{\prime}-2 \tau^{*} H\right)^{\oplus a+c} .
\end{aligned}
$$

\footnotetext{
3 The referee pointed out that this and the next congruences of binomial coefficients follow from Lucas' theorem; see, e.g., [F].
} 
Comparing this decomposition with that in Lemma 1.3, we obtain $a=q$, $b=0$ and $c=q(q-1) / 2$. The formulas for $F_{*}^{e} \mathcal{B}$ and $F_{*}^{e} \mathcal{G}$ follow similarly from Theorems 4.1, 5.1 and Lemma 1.3.

\section{Concluding remarks and questions}

We are now at the starting point of the following.

QUESTION 6.1. Let $X$ be the blowup of $\mathbb{P}^{2}$ at $n$ points in general position. Does there exist an upper bound of $n$ for which $X$ is GFFRT? If it does, what is the effective bound?

Here is a positive evidence for the existence of an upper bound.

Proposition 6.2. Let $C$ be an elliptic curve on $\mathbb{P}^{2}$ and let $P_{1}, \ldots, P_{10}$ be distinct ten points on $C$. Assume that $C$ and $P_{1}, \ldots, P_{10}$ are defined over the algebraic closure $\overline{\mathbb{F}_{p}}$ of the prime field. Let $X$ be the rational surface obtained by blowing up $\mathbb{P}^{2}$ at the points $P_{1}, \ldots, P_{10}$. Then $X$ is not GFFRT.

Proof. Let $\pi: X \rightarrow \mathbb{P}^{2}$ be the blowup morphism and let $\widetilde{C}$ be the strict transform of $C$ with respect to $\pi$. Then $X$ and $\widetilde{C}$ are defined over $\overline{\mathbb{F}_{p}}$ and $\widetilde{C}^{2}=-1<0$. We choose a very ample divisor $A$ on $X$ (also defined over $\overline{\mathbb{F}_{p}}$ ) and let $L=A+(A \cdot \widetilde{C}) \widetilde{C}$. Then $L$ is a nef and big line bundle such that $L \widetilde{C}=0$ and $L D>0$ for any reduced curve $D \neq \widetilde{C}$. Since everything involving is defined over $\overline{\mathbb{F}_{p}}$, it follows from $[\mathrm{K}$, Corollary 0.3$]$ that $L$ is semi-ample. Thus, we can choose a positive integer $m$ such that $m L$ gives a morphism $\Phi_{|m L|}: X \rightarrow \mathbb{P}^{h^{0}(m L)-1}$ birational onto its image $X^{\prime}=\Phi_{|m L|}(X)$. Denoting the normalization of $X^{\prime}$ by $Y$, we obtain a birational morphism $\varphi: X \rightarrow Y$ via which $\widetilde{C}$ contracts to a simple elliptic singularity $(Y, y)$.

In what follows, we will employ $F$-blowup introduced by Yasuda $[\mathrm{Y}]$ in order to avoid an argument involving completion of the local ring $\mathcal{O}_{Y, y}$; cf. [Y, Proposition 2.11]. It is easy to observe the following.

(1) If $X$ is GFFRT, then the singularity $(Y, y)$ is FFRT as defined in [SVdB], that is, there exists a finite set of $\mathcal{O}_{Y, y}$-modules such that for every $e \geq 0$, $F_{*}^{e}\left(\mathcal{O}_{Y}\right)_{y} \cong \mathcal{O}_{Y, y}^{1 / p^{e}}$ is isomorphic to a direct sum of copies of those finitely many modules.

(2) If $(Y, y)$ is FFRT, then the $F$-blowup sequence $\left\{\mathrm{FB}_{e}(Y) \mid e=1,2, \ldots\right\}$ of $(Y, y)$ stabilizes.

However, it follows from [HSY] that the F-blowup sequence of the simple elliptic singularity $(Y, y)$ of type $\widetilde{E}_{8}$ does not stabilize in general. Thus, $X$ is not GFFRT.

REMARK 6.3. In the proposition above, if $C$ is an ordinary elliptic curve, then $X$ is $F$-split. On the other hand, there is a notion of global $F$-regularity 
[Sm], which is stronger than $F$-splitting, and we do not have an example of a globally $F$-regular surface that is not GFFRT.

6.1. Interpretation via derived category. We interpret the structure of the Frobenius push-forward $F_{*}^{e} \mathcal{O}_{X}$ from a viewpoint of derived category. For a smooth projective variety $X$, we denote by $D^{b}(X)$ the bounded derived category of coherent sheaves on $X$. A coherent sheaf $\mathcal{F}$ on $X$ is said to be exceptional if $\operatorname{Hom}(\mathcal{F}, \mathcal{F})=k$ and $\operatorname{Ext}^{i}(\mathcal{F}, \mathcal{F})=0$ for all $i \neq 0$. An ordered collection of exceptional sheaves $\mathcal{F}_{1}, \ldots, \mathcal{F}_{n}$ is called an exceptional collection if $\operatorname{Ext}^{i}\left(\mathcal{F}_{k}, \mathcal{F}_{j}\right)=0$ for all integers $i$ and for $1 \leq j<k \leq n$. An exceptional collection $\mathcal{F}_{1}, \ldots, \mathcal{F}_{n}$ is said to be strong if $\operatorname{Ext}^{i}\left(\mathcal{F}_{j}, \mathcal{F}_{k}\right)=0$ for $i>0$ and $1 \leq j, k \leq n$. An exceptional collection is said to be full if it generates the derived category $D^{b}(X)$.

It turns out that if $p^{e} \geq 3$, then the $e$ th Frobenius push-forward $F_{*}^{e} \mathcal{O}_{X}$ on the four-point blowup $X$ of $\mathbb{P}^{2}$ generates the derived category $D^{b}(X)$ in the same sense as in Ohkawa-Uehara [OU], where results in the toric case are established. ${ }^{4}$

Proposition 6.4. Let $X$ be the blowup of $\mathbb{P}^{2}$ at four points in general position and let the notation be as in Section 5.1. Then Frobenius summands $\mathcal{G}, L_{0}, L_{1}, L_{2}, L_{3}, L_{4}, \mathcal{O}_{X}$ on $X$ form a full strong exceptional collection.

Proof. First, we will show that $\mathcal{G}, L_{0}, L_{1}, L_{2}, L_{3}, L_{4}, \mathcal{O}_{X}$ is an exceptional collection. We know that any line bundle on $X$ is exceptional, $\operatorname{Ext}^{i}\left(\mathcal{O}_{X}, \mathcal{F}\right)=$ 0 for any $i \in \mathbb{Z}$ and any indecomposable Frobenius summand $\mathcal{F} \not \mathcal{O}_{X}$, and that $\operatorname{Ext}^{i}\left(L_{j}, L_{k}\right)=0$ for any $i$ and $0 \leq j, k \leq 4$ with $j \neq k$; cf. Proposition 3.4. Thus, it remains to show that $\mathcal{G}$ is exceptional and $\operatorname{Ext}^{i}\left(L_{j}, \mathcal{G}\right)=0$ for all $i$ and $0 \leq j \leq 4$. To this end, we look at an Ext long exact sequence induced by the non-trivial extension (3.2):

$$
\begin{aligned}
& 0 \rightarrow \operatorname{Hom}\left(L_{0}, \mathcal{O}_{X}\left(-\pi^{*} H\right)\right) \rightarrow \operatorname{Hom}\left(L_{0}, \mathcal{G}\right) \rightarrow \operatorname{Hom}\left(L_{0}, L_{0}\right) \\
& \quad \stackrel{\delta}{\rightarrow} \operatorname{Ext}^{1}\left(L_{0}, \mathcal{O}_{X}\left(-\pi^{*} H\right)\right) \rightarrow \operatorname{Ext}^{1}\left(L_{0}, \mathcal{G}\right) \rightarrow \cdots .
\end{aligned}
$$

Since $\operatorname{Hom}\left(L_{0}, \mathcal{O}_{X}\left(-\pi^{*} H\right)\right)=\operatorname{Ext}^{2}\left(L_{0}, \mathcal{O}_{X}\left(-\pi^{*} H\right)\right)=\operatorname{Ext}^{i}\left(L_{0}, L_{0}\right)=0$ for $i \geq 1$ and $\delta$ is an isomorphism of 1 -dimensional vector spaces, we see that $\operatorname{Ext}^{i}\left(L_{0}, \mathcal{G}\right)=0$ for all $i \in \mathbb{Z}$. This vanishing, together with a suitable Cremona automorphism, implies that $\operatorname{Ext}^{i}\left(L_{j}, \mathcal{G}\right)=0$ holds for all $i$ and $0 \leq j \leq 4$; cf. Example 3.1. Next, we note that $\operatorname{Ext}^{i}\left(\mathcal{O}_{X}\left(-\pi^{*} H\right), L_{0}\right)=$ $H^{i}\left(X, \mathcal{O}_{X}\left(E-\pi^{*} H\right)\right)=0$ for all $i$ by Riemann-Roch and look at the long exact sequence of $\operatorname{Ext}\left(\mathcal{O}_{X}\left(-\pi^{*} H\right),-\right)$ applied to (3.2). It then follows that $\operatorname{Hom}\left(\mathcal{O}_{X}\left(-\pi^{*} H\right), \mathcal{G}\right) \cong k$ and $\operatorname{Ext}^{i}\left(\mathcal{O}_{X}\left(-\pi^{*} H\right), \mathcal{G}\right)=0$ for $i \geq 1$. Now looking

\footnotetext{
4 Actually, the result on the generation of the derived category by the Frobenius pushforward is extended to the case of two-dimensional toric stacks as the title of the paper $[\mathrm{OU}]$ indicates.
} 
at the long exact sequence of $\operatorname{Ext}(-, \mathcal{G})$ applied to $(3.2)$ we conclude that $\operatorname{Hom}(\mathcal{G}, \mathcal{G})=k$ and $\operatorname{Ext}^{i}(\mathcal{G}, \mathcal{G})=0$ for $i \geq 1$, i.e., $\mathcal{G}$ is exceptional.

We can verify similarly as above that $\operatorname{Ext}^{i}\left(L, \mathcal{O}_{X}\right)=\operatorname{Ext}^{i}(\mathcal{G}, L)=0$ for all $i>0$ and $L=\mathcal{O}_{X}$ or $L_{j}$ with $0 \leq j \leq 4$. Thus, the exceptional sequence is strong.

To prove that the exceptional collection is full, let $\mathcal{D}=\left\langle\mathcal{G}, L_{i}, \mathcal{O}_{X} \mid 0 \leq i \leq 4\right\rangle$ be the subcategory of $D^{b}(X)$ generated by $\mathcal{G}, L_{0}=\mathcal{O}_{X}\left(E-2 \pi^{*} H\right), L_{i}=$ $\mathcal{O}_{X}\left(E_{i}-\pi^{*} H\right)(i=1,2,3,4)$ and $\mathcal{O}_{X}$. Since $\mathcal{G}$ and $L_{0}$ are objects of $\mathcal{D}$, it follows from $(3.2)$ that $\mathcal{O}_{X}\left(-\pi^{*} H\right)$ is an object of $\mathcal{D}$. It then follows from the exact sequence

$$
0 \rightarrow \mathcal{O}_{X}\left(-\pi^{*} H\right) \rightarrow \mathcal{O}_{X}\left(E_{i}-\pi^{*} H\right) \rightarrow \mathcal{O}_{E_{i}}(-1) \rightarrow 0
$$

that $\mathcal{O}_{E_{i}}(-1)$ is an object of $\mathcal{D}$ for $i=1,2,3,4$. Finally, it follows from the exact sequence

$$
0 \rightarrow \mathcal{O}_{X}\left(-2 \pi^{*} H\right) \rightarrow \mathcal{O}_{X}\left(E-2 \pi^{*} H\right) \rightarrow \bigoplus_{i=1}^{4} \mathcal{O}_{E_{i}}(-1) \rightarrow 0
$$

that $\mathcal{O}_{X}\left(-2 \pi^{*} H\right)$ is an object of $\mathcal{D}$. Since $D^{b}\left(\mathbb{P}^{2}\right)=\left\langle\mathcal{O}_{\mathbb{P}^{2}}(-2), \mathcal{O}_{\mathbb{P}^{2}}(-1), \mathcal{O}_{\mathbb{P}^{2}}\right\rangle$ and $D^{b}(X)=\left\langle\pi^{*} D^{b}\left(\mathbb{P}^{2}\right), \mathcal{O}_{E_{i}}(-1) \mid 1 \leq i \leq 4\right\rangle$ by [Hu, Proposition 11.18], we conclude that $\mathcal{D}=D^{b}(X)$, that is, the exceptional collection is full.

Finally, we ask the following optimistic questions.

Question 6.5. Let $X$ be a smooth globally $F$-regular surface.

(1) Is $X$ GFFRT?

(2) Is $D^{b}(X)$ generated by Frobenius summands on $X$ ?

Acknowledgments. I would like to thank Shigeru Mukai for pointing out an error in an earlier version of this note. I am grateful to Ryo Ohkawa, Masahiro Ohno and Akiyoshi Sannai for invaluable discussions and suggestive comments. I also thank the referee for careful reading and helpful comments.

\section{REFERENCES}

[A1] P. Achinger, Frobenius push-forwards on quadrics, Comm. Algebra 40 (2012), no. 8, 2732-2748. MR 2968908

[A2] P. Achinger, A characterization of toric varieties in characteristic $p$, available at arXiv: 1303.5905.

[At] M. F. Atiyah, Vector bundles over an elliptic curve, Proc. Lond. Math. Soc. (3) 7 (1957), 414-452. MR 0131423

[F] N. J. Fine, Binomial coefficients modulo a prime, Amer. Math. Monthly 54 (1947), no. 10, 589-592. MR 0023257

$[\mathrm{H}] \quad$ N. Hara, A characterization of rational singularities in terms of injectivity of Frobenius maps, Amer. J. Math. 120 (1998), 981-996. MR 1646049

[HSY] N. Hara, T. Sawada and T. Yasuda, F-blowups of normal surface singularities, Algebra Number Theory 7 (2013), 733-763. MR 3095225 
[Hu] D. Huybrechts, Fourier-Mukai transforms in algebraic geometry, Oxford Mathematical Monographs, The Clarendon Press, Oxford University Press, Oxford, 2006. MR 2244106

[K] S. Keel, Basepoint freeness for nef and big line bundles in positive characteristic, Ann. of Math. (2) 149 (1999), 253-286. MR 1680559

[KS] Y. Kitadai and H. Sumihiro, Canonical filtrations and stability of direct images by Frobenius morphisms, Tohoku Math. J. (2) 60 (2008), 287-301. MR 2428865

[MP] V. B. Mehta and C. Pauly, Semistability of Frobenius direct images over curves, Bull. Soc. Math. France 135 (2007), 105-117. MR 2430201

[OU] R. Ohkawa and H. Uehara, Frobenius morphisms and derived categories on twodimensional toric Deligne-Mumford stacks, Adv. Math. 244 (2013), 241-267. MR 3077872

[ST] A. Sannai and H. Tanaka, A characterization of ordinary abelian varieties by the Frobenius push-forward of the structure sheaf, preprint; available at arXiv: 1411.5294.

[Sch] K. Schwede, F-adjunction, Algebra Number Theory 3 (2009), 907-950. MR 2587408

[Sm] K. E. Smith, Globally F-regular varieties: Applications to vanishing theorems for quotients of Fano varieties, Michigan Math. J. 48 (2000), 553-572. MR 1786505

[SVdB] K. E. Smith and M. Van den Bergh, Simplicity of rings of differential operators in prime characteristic, Proc. Lond. Math. Soc. (3) 75 (1997), 32-62. MR 1444312

[Su] X. Sun, Direct images of bundles under Frobenius morphism, Invent. Math. $\mathbf{1 7 3}$ (2008), 427-447. MR 2415312

[T] J. F. Thomsen, Frobenius direct images of line bundles on toric varieties, J. Algebra 226 (2000), 865-874. MR 1752764

[Y] T. Yasuda, Universal flattening of Frobenius, Amer. J. Math. 134 (2012), 349-378. MR 2905000

Nobuo Hara, Tokyo University of Agriculture and Technology, 2-24-16 NakaCho, Koganei, TOKYo, 184-8588, JAPAn

E-mail address: nhara@cc.tuat.ac.jp 